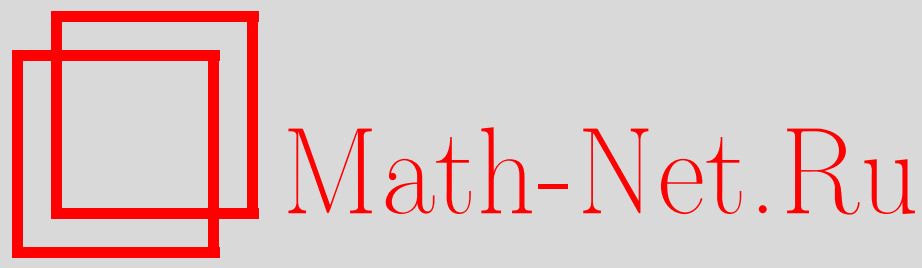

К. А. Попков, О единичных тестах для функциональных элементов, Дискрет. матем., 2015, том 27, выпуск 2, 7393

DOI: https://doi.org/10.4213/dm1326

Использование Общероссийского математического портала Math-Net.Ru подразумевает, что вы прочитали и согласны с пользовательским соглашением http://www . mathnet.ru/rus/agreement

Параметры загрузки:

IP : 54.198 .187 .58

26 апреля 2023 г., 18:23:42

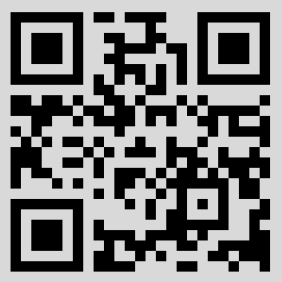




\title{
О единичных тестах для функциональных элементов
}

\author{
(c) 2015 г. K. A. Попков*
}

\begin{abstract}
Рассматриваются задачи проверки исправности и диагностики состояний $N$ функциональных элементов, реализующих в исправном состоянии заданную булеву функцию $f\left(x_{1}, \ldots, x_{n}\right)$, путём составления из них схем с одним выходом и анализа выдаваемых этими схемами значений на любых входных наборах значений переменных. Допускается произвольная константная неисправность на выходе любого одного функционального элемента. Требуется минимизировать число схем, необходимых для проверки исправности и определения состояний всех элементов. В работе получены точные значения для минимально возможного числа указанных схем.
\end{abstract}

Ключевые слова: функциональный элемент, неисправность, схема, проверяющий тест, диагностический тест.

\section{1. Введение}

В работе рассматриваются задачи проверки исправности и распознавания состояний функциональных элементов с использованием экспериментов, заключающихся в составлении произвольных схем [1] из заданных функциональных элементов с последующим "прозваниванием" этих схем, т. е. нахождением булевых функций, реализуемых составляемыми схемами. Постановка указанных задач описана в [2]. Всюду в данной работе предполагается, что среди рассматриваемых функциональных элементов неисправным может оказаться лишь один.

Будем предполагать, что для любого $i$ от 1 до $N$ элемент $E_{i}$ имеет номер $i$.

Неисправностъю системы элементов будем называть любое множество неисправностей заданных функциональных элементов при условии, что число неисправных элементов не больше одного. (В частности, случай, когда все элементы исправны, является одним из видов неисправности системы элементов.)

Неисправность любого элемента можно представить в виде упорядоченной пары $(i, \delta)$, где $i$ - номер этого элемента, $\delta$ - булева константа, которую он реализует. Соответственно, любую неисправность системы элементов можно представить в виде множества $\{(i, \delta)\}$, если функциональный элемент с номером $i$ неисправен и реализует константу $\delta$, либо пустого множества, если все $N$ функциональных элементов исправны.

Диагностическим тестом назовём такой набор схем $S_{1}, \ldots, S_{l}$ с одним выходом, составленных из заданных функциональных элементов, что для любых двух различных неисправностей системы элементов наборы функций, реализуемых схемами, не

* Место работы: МГУ им. М. В. Ломоносова, e-mail: kirill-formulist@mail.ru 
совпадают (т. е. существует такая схема $S_{j}$, что реализуемая этой схемой функция при первой неисправности не совпадает с реализуемой этой же схемой функцией при второй неисправности). Число $l$ назовём длиной этого теста.

Проверяющим тестом назовём такой набор схем $S_{1}, \ldots, S_{l}$ с одним выходом, составленных из заданных функциональных элементов, что для любых двух неисправностей системы элементов, при которых множества неисправных элементов различны, наборы функций, реализуемых схемами, не совпадают. Число $l$ назовём длиной этого теста.

Содержательный смысл данных определений состоит в следующем: диагностический (проверяющий) тест - это такой набор схем $S_{1}, \ldots, S_{l}$ с одним выходом, составленных из заданных $N$ функциональных элементов, что по набору функций, реализуемых этими схемами, можно однозначно определить состояние (соответственно, исправность или неисправность) каждого из $N$ элементов. При этом проверяющий тест не обязан определять тип неисправности (0 или 1) неисправного элемента, если такой элемент существует.

Введём функции $L_{c}(f, N)$ и $L_{d}(f, N)$, равные длинам самого короткого, соответственно, проверяющего и диагностического тестов для $N$ функциональных элементов, среди которых не более одного элемента неисправно (в исправном состоянии каждый элемент реализует булеву функцию $f$ ).

Отметим, что введённые определения диагностического и проверяющего тестов и функций $L_{c}(f, N), L_{d}(f, N)$ совпадают с определениями соответственно диагностического и проверяющего тестов и функций $L_{c}(f, N, k), L_{d}(f, N, k)$ из [2] при $k=1$.

Поскольку в работе рассматриваются только единичные неисправности функциональных элементов, диагностический (проверяющий) тест будем также называть единичным диагностическим (проверяющим) тестом.

Утверждение 1. Множества единичных проверяющих и единичных диагностических тестов для $N$ функииональных элементов, каждый из которых реализует в исправном состоянии булеву функцию $f$, совпадают.

Следствие 1. Справедливо равенство $L_{c}(f, N)=L_{d}(f, N)$.

Доказательство утверждения 1. Из определений следует, что любой диагностический тест является проверяющим; докажем обратное утверждение. Пусть $\left(S_{1}, \ldots, S_{l}\right)$ - проверяющий тест. Достаточно доказать, что для любого $i \in$ $\{1, \ldots, N\}$ для неисправностей $H_{0}=\{(i, 0)\}$ и $H_{1}=\{(i, 1)\}$ системы элементов наборы функций, реализуемых схемами $S_{1}, \ldots, S_{l}$, не совпадают. Рассмотрим неисправность системы элементов $H=\varnothing$. Так как элемент $E_{i}$ исправен при $H$ и неисправен при $H_{0}$, а $\left(S_{1}, \ldots, S_{l}\right)$ - проверяющий тест, то наборы функций, реализуемых схемами $S_{1}, \ldots, S_{l}$ при неисправностях $H$ и $H_{0}$, не совпадают. Это означает, что функции, реализуемые некоторой схемой $S$ из $S_{1}, \ldots, S_{l}$ при неисправностях $H$ и $H_{0}$, различаются. Обозначим эти функции соответственно через $f_{S, H}$ и $f_{S, H_{0}}$, а функцию, реализуемую схемой $S$ при неисправности $H_{1}$ - через $f_{S, H_{1}}$. Тогда существует такой набор $\tilde{\alpha}$ значений входных переменных схемы $S$, что

$$
f_{S, H}(\tilde{\alpha}) \neq f_{S, H_{0}}(\tilde{\alpha}) \text {. }
$$

Отсюда следует, что при неисправности $H$ на входном наборе $\tilde{\alpha}$ значение на выходе элемента $E_{i}$ в схеме $S$ равно 1 , так как в случае равенства этого значения нулю 
изменение состояния элемента $E_{i}$ с исправного на неисправное типа 0 , т. е. переход от неисправности $H$ к неисправности $H_{0}$, никак не отразилось бы на значении, выдаваемом схемой $S$ на входном наборе $\tilde{\alpha}$, что невозможно в силу (1). Но тогда изменение состояния элемента $E_{i}$ с исправного на неисправное типа 1 , т. е. переход от неисправности $H$ к неисправности $H_{1}$, никак не отразится на значении, выдаваемом схемой $S$ на входном наборе $\tilde{\alpha}$, т. е.

$$
f_{S, H}(\tilde{\alpha})=f_{S, H_{1}}(\tilde{\alpha})
$$

Из (1) и (2) следует, что $f_{S, H_{1}}(\tilde{\alpha}) \neq f_{S, H_{0}}(\tilde{\alpha})$, откуда заключаем, что функции $f_{S, H_{0}}$ и $f_{S, H_{1}}$ не совпадают. Следовательно, наборы функций, реализуемых схемами $S_{1}, \ldots, S_{l}$ при неисправностях $H_{0}$ и $H_{1}$, не совпадают. Утверждение 1 доказано.

Замечание 1. В силу следствия из утверждения 1 для нахождения величины $L_{d}(f, N)$ достаточно знать только $L_{c}(f, N)$. Поэтому после введения обозначения $L(f, N)=L_{c}(f, N)$ можно формулировать основные результаты в терминах величины $L(f, N)$.

\section{2. Формулировка и доказательство основного резуль- тата}

Напомним, что согласно предположениям, сделанным в постановке задачи в [2], функция $f\left(x_{1}, \ldots, x_{n}\right)$ существенно зависит от всех своих $n$ переменных, где $n \geqslant 1$. Выделим два возможных типа функции $f$ :

$$
\begin{aligned}
& f\left(x_{1}, \ldots, x_{n}\right)=x_{1}^{\sigma_{1}} \& \ldots \& x_{n}^{\sigma_{n}}, \\
& f\left(x_{1}, \ldots, x_{n}\right)=x_{1}^{\sigma_{1}} \vee \ldots \vee x_{n}^{\sigma_{n}},
\end{aligned}
$$

где $\sigma_{1}, \ldots, \sigma_{n} \in\{0,1\}$.

Основным результатом данной работы является следующая теорема.

Теорема 1. Справедливо равенство

$$
L(f, N)=\left\{\begin{array}{l}
1, \text { если функция } f \text { не представима ни в одном из видов }(*),(* *) ; \\
\min (2 ; N), \text { если функция } f \text { представима в виде }(*) \text { или }(* *), \\
\quad \text { рричём } n \geqslant 2 \text { и хотя би одно из чисел } \sigma_{1}, \ldots, \sigma_{n} \text { равно нулю; } \\
\left\lceil\log _{2}(N+1)\right\rceil, \text { если } f\left(x_{1}, \ldots, x_{n}\right) \in\left\{x_{1} \& \ldots \& x_{n}, x_{1} \vee \ldots \vee x_{n}\right\} ; \\
\left\lceil\log _{3}(2 N+1)\right\rceil, \text { если } n=1 \text { и } f\left(x_{1}\right)=\overline{x_{1}} .
\end{array}\right.
$$

Замечание 2. Легко видеть, что четыре случая, указанные в формулировке основной теоремы, охватывают все возможные случаи.

Доказательство основной теоремы. Для удобства пронумеруем указанные четыре случая цифрами 1-4 в том порядке, в каком они идут в формулировке теоремы. Тогда соотношение $L(f, N)=1$ в случае 1 следует из теоремы 2 из [2] при $k=1$. Оценка $L(f, N) \geqslant \min (2 ; N)$ в случае 2 при $N \geqslant 2$ следует из теоремы 3 из [2] при $m=n-1$ и $k=1$ и тривиальна при $N=1$ (пустой набор схем не может быть проверяющим тестом). Оценки $L(f, N) \geqslant\left\lceil\log _{2}(N+1)\right\rceil$ в случае 3 и 
$L(f, N) \geqslant\left\lceil\log _{3}(2 N+1)\right\rceil$ в случае 4 следуют из теорем 1 и 2 из [3] при $k=1$ соответственно. Таким образом, достаточно получить оценки $L(f, N) \leqslant \min (2 ; N)$ в случае $2, L(f, N) \leqslant\left\lceil\log _{2}(N+1)\right\rceil$ в случае 3 и $L(f, N) \leqslant\left\lceil\log _{3}(2 N+1)\right\rceil$ в случае 4 .

Рассмотрим случай 2. Если $N=1$, то схема, состоящая из единственного элемента, на входы которого подаются переменные $x_{1}, \ldots, x_{n}$, очевидно, составляет проверяющий тест длины 1 , поэтому $L(f, N) \leqslant 1=\min (2 ; N)$. Пусть

$$
N \geqslant 2 \text {. }
$$

По предположению случая 2 функция $f$ представима в виде $(*)$ или $(* *)$, причём $n \geqslant 2$ и хотя бы одно из чисел $\sigma_{1}, \ldots, \sigma_{n}$ равно нулю. Без ограничения общности будем считать, что

$$
\sigma_{1}=0
$$

(в противном случае можно соответствующим образом перенумеровать входы каждого элемента). Построим схему $S_{1}$ следующим образом. Пусть для каждого $s=1, \ldots, N$ на входы $v_{2}, \ldots, v_{n}$ элемента $E_{s}$ подаются соответственно переменные $x_{s, 2}, \ldots, x_{s, n}$, а на его вход $v_{1}$ либо подаётся переменная $x_{1,1}$, если $s=1$, либо этот вход соединяется с выходом элемента $E_{s-1}$, если $s \geqslant 2$. Выход элемента $E_{N}$ будем считать выходом схемы $S_{1}$. Далее построим схему $S_{2}$ следующим образом. Пусть для каждого $s=1, \ldots,\left\lfloor\frac{N}{2}\right\rfloor$ на входы $v_{2}, \ldots, v_{n}$ элемента $E_{2 s}$ подаются соответственно переменные $x_{s, 2}, \ldots, x_{s, n}$, а на его вход $v_{1}$ либо подаётся переменная $x_{1,1}$, если $s=1$, либо этот вход соединяется с выходом элемента $E_{2 s-2}$, если $s \geqslant 2$. Выход элемента $E_{2\left\lfloor\frac{N}{2}\right\rfloor}$ будем считать выходом схемы $S_{2}$; схемы $S_{1}, S_{2}$ показаны на рис. 1 .

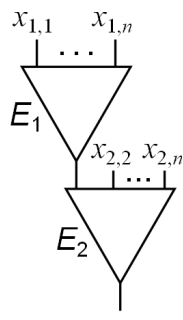

$\cdots$

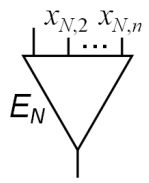

$S_{1}$
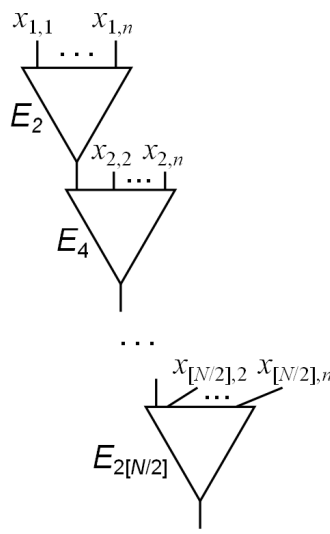

$S_{2}$

Рис. 1

Ограничимся рассмотрением случая, когда функция $f$ имеет вид $\left(^{*}\right)$ (случай, когда $f$ имеет вид $(* *)$, рассматривается двойственным образом).

Лемма 1. Пусть $\mathrm{H}_{1}$ и $\mathrm{H}_{2}$ - две такие неисправности системы элементов, при которых множества неисправных элементов различны, а функиии, реализуемые 
схемой $S_{1}$, совпадают. Тогда при $H_{1}$ неисправен некоторый элемент $E_{i_{1}}$, а при $H_{2}$ - некоторый элемент $E_{i_{2}}$, причём $\left|i_{1}-i_{2}\right|=1$.

Доказательство. Пусть $g-$ функция, реализуемая схемой $S_{1}$ при неисправности $H_{1}$ (а значит, и при $H_{2}$ ). Предположим сначала, что при одной из неисправностей $H_{1}$ и $H_{2}$ (без ограничения общности, при $H_{1}$ ) все элементы исправны. Тогда с учётом построения схемы $S_{1}$ и существенной зависимости функции $f\left(x_{1}, \ldots, x_{n}\right)$ от всех своих переменных нетрудно заметить, что функция $g$ существенно зависит от переменной $x_{1,1}$. С другой стороны, в силу условия леммы, при неисправности $H_{2}$ некоторый элемент обязан быть неисправным и реализовывать константу. Этот элемент, очевидно, содержится в единственной цепи, связывающей вход " $x_{1,1}$ " схемы $S_{1}$ (отвечающий переменной $x_{1,1}$ ) с её выходом. Отсюда заключаем, что функция $g$, реализуемая схемой $S_{1}$ при неисправности $H_{2}$, не может зависеть существенно от переменной $x_{1,1}$. Получено противоречие, следовательно, исходное предположение неверно и при неисправности $H_{1}$ неисправен некоторый элемент $E_{i_{1}}$, а при $H_{2}-$ некоторый элемент $E_{i_{2}}$. Из условия леммы следует, что $i_{1} \neq i_{2}$. Предположим, что $\left|i_{1}-i_{2}\right| \geqslant 2$. Без ограничения общности, $i_{1}>i_{2}$, а тогда

$$
i_{1}-i_{2} \geqslant 2 \text {. }
$$

По построению схемы $S_{1}$ единственная цепь, связывающая вход " $x_{i_{1}, 2}$ " этой схемы с её выходом, проходит через элемент $E_{i_{1}}$. При неисправности $H_{1}$ данный элемент неисправен и реализует константу, откуда заключаем, что функция $g$ не может зависеть существенно от переменной $x_{i_{1}, 2}$. С другой стороны, пусть имеет место неисправность $H_{2}$. Тогда в силу (5) $i_{1}-1 \geqslant i_{2}+1>i_{2}$, откуда следует, что элемент $E_{i_{1}-1}$ и все элементы с номерами, большими $i_{1}-1$, исправны. Предположим, что на вход схемы $S_{1}$ вместо переменной $x_{i_{1}-1,2}$ подано значение $\overline{\sigma_{2}}$. Тогда на вход $v_{2}$ элемента $E_{i_{1}-1}$ в схеме $S_{1}$ подаётся константа $\overline{\sigma_{2}}$ и вне зависимости от того, какие функции подаются на остальные входы этого элемента, на его выходе будет реализована функция $\ldots \&{\overline{\sigma_{2}}}^{\sigma_{2}} \& \ldots=0$ в силу того, что функция $f$ имеет вид $(*)$, а элемент $E_{i_{1}-1}$ исправен. В таком случае на вход $v_{1}$ элемента $E_{i_{1}}$ будет подаваться константа 0 , и функция, реализуемая на выходе этого элемента, в силу (4) и исправности элемента $E_{i_{1}}$ будет равна $f\left(0, x_{i_{1}, 2} \ldots, x_{i_{1}, n}\right)=0^{\sigma_{1}} \& x_{i_{1}, 2}^{\sigma_{2}} \& \ldots \& x_{i_{1}, n}^{\sigma_{n}}=x_{i_{1}, 2}^{\sigma_{2}} \& \ldots \& x_{i_{1}, n}^{\sigma_{n}}$. Отсюда с учётом построения схемы $S_{1}$, существенной зависимости функции $f\left(x_{1}, \ldots, x_{n}\right)$ от всех своих переменных и исправности всех элементов с номерами, большими $i_{1}$ (если такие есть) нетрудно вывести, что функция, реализуемая схемой $S_{1}$ при неисправности $H_{2}$, будет существенно зависеть от переменной $x_{i_{1}, 2}$. Это означает, что и исходная функция, реализуемая схемой $S_{1}$ при неисправности $H_{2}$ (до подачи вместо переменной $x_{i_{1}-1,2}$ значения $\overline{\sigma_{2}}$ ), т. е. функция $g$, существенно зависит от переменной $x_{i_{1}, 2}$, однако ранее было показано, что это невозможно. Полученное противоречие доказывает равенство $\left|i_{1}-i_{2}\right|=1$, а вместе с ним лемму 1.

Лемма 2. Набор $\left(S_{1}, S_{2}\right)$ является проверяющим тестом.

Доказательство. Пусть $H_{1}$ и $H_{2}$ - две произвольные неисправности системы элементов, при которых множества неисправных элементов различны. Надо доказать, что наборы функций, реализуемых схемами $S_{1}, S_{2}$ при этих двух неисправностях, не совпадают. Если схема $S_{1}$ реализует при $H_{1}$ и $H_{2}$ различные функции, то утверждение доказано. Пусть данная схема реализует при этих неисправностях одну и ту же функцию. Тогда выполнены все условия леммы 1 , из которой заключаем, что при $H_{1}$ неисправен некоторый элемент $E_{i_{1}}$, а при $H_{2}$ - некоторый элемент $E_{i_{2}}$, причём 
$\left|i_{1}-i_{2}\right|=1$. Из последнего равенства следует, что ровно одно из чисел $i_{1}, i_{2}$ (без ограничения общности, $\left.i_{1}\right)$ чётно. По построению схемы $S_{2}$ в единственной цепи, связывающей вход " $x_{1,1}$ " этой схемы с её выходом, содержатся все элементы с чётными номерами, в том числе и элемент $E_{i_{1}}$, который при неисправности $H_{1}$ неисправен и реализует константу. Это означает, что функция, реализуемая на выходе схемы $S_{2}$ при неисправности $H_{1}$, не может зависеть существенно от переменной $x_{1,1}$. С другой стороны, пусть имеет место неисправность $H_{2}$. По построению схемы $S_{2}$ в ней не содержится ни одного элемента с нечётным номером, в том числе элемента $E_{i_{2}}$, так как $i_{2}$ нечётно. Но $E_{i_{2}}$ - единственный неисправный элемент при неисправности $H_{2}$, откуда следует, что все элементы, содержащиеся в схеме $S_{2}$, исправны. Тогда с учётом построения схемы $S_{2}$ и существенной зависимости функции $f\left(x_{1}, \ldots, x_{n}\right)$ от всех своих переменных нетрудно заметить, что функция, реализуемая на выходе схемы $S_{2}$, существенно зависит от переменной $x_{1,1}$. В итоге получаем, что на выходе этой схемы при неисправностях $H_{1}$ и $H_{2}$ реализуются различные функции (одна из них зависит существенно от переменной $x_{1,1}$, а другая - нет), а значит, наборы функций, реализуемых схемами $S_{1}, S_{2}$ при этих двух неисправностях, не совпадают. Лемма 2 доказана.

Из леммы 2 и (3) получаем соотношение $L(f, N) \leqslant 2=\min (2 ; N)$. Случай 2 разобран.

Рассмотрим теперь случай 3 , т. е. $f\left(x_{1}, \ldots, x_{n}\right) \in\left\{x_{1} \& \ldots \& x_{n}, x_{1} \vee \ldots \vee x_{n}\right\}$. Введём обозначение $m=\left\lceil\log _{2}(N+1)\right\rceil$. Для каждого $j$ от 1 до $m$ построим схему $S_{j}^{\prime}$ следующим образом. Пусть $M_{j}-$ множество таких заданных функциональных элементов, в $j$-м справа разряде двоичной записи номера которых стоит единица. Например, множество $M_{1}$ состоит из всех элементов с нечётными номерами. Отметим, что множество $M_{j}$ не пусто, так как в нём содержится элемент $E_{2^{j-1}}$. Действительно, в $j$-м справа разряде двоичной записи числа $2^{j-1}$ стоит единица; с другой стороны, $1 \leqslant 2^{j-1} \leqslant 2^{m-1}=2^{\left\lceil\log _{2}(N+1)\right\rceil-1}<2^{\log _{2}(N+1)}=N+1$, откуда следует, что $1 \leqslant 2^{j-1} \leqslant N$ и элемент $E_{2^{j-1}}$ существует. Возьмём любой элемент из множества $M_{j}$ и подадим на все его $n$ входов переменную $x$. Далее, возьмём любой другой элемент из множества $M_{j}$ (если такой элемент существует) и соединим все его $n$ входов с выходом предыдущего элемента. Затем возьмём любой элемент из множества $M_{j}$, отличный от первых двух (если такой элемент существует) и соединим все его $n$ входов с выходом предыдущего элемента, и т.д. В итоге получим цепь, содержащую все элементы из множества $M_{j}$. Выход нижнего элемента этой цепи будем считать выходом схемы $S_{j}^{\prime}$. Из вида этой схемы и того, что $f\left(x_{1}, \ldots, x_{n}\right) \in\left\{x_{1} \& \ldots \& x_{n}, x_{1} \vee \ldots \vee x_{n}\right\}$, непосредственно вытекает следующее утверждение.

Лемма 3. Пусть имеет место некоторая неисправность системы элементов. Тогда функиия, реализуемая схемой $S_{j}^{\prime}$, равна

$$
\left\{\begin{array}{l}
x, \quad \text { если все элементы в схеме } S_{j}^{\prime} \text { исправны; } \\
0, \quad \text { если некоторый элемент в схеме } S_{j}^{\prime} \text { неисправен и выдаёт } 0 ; \\
1, \quad \text { если некоторый элемент в схеме } S_{j}^{\prime} \text { неисправен и выдаёт } 1 .
\end{array}\right.
$$

В итоге построены схемы $S_{1}^{\prime}, \ldots, S_{m}^{\prime}$. Отметим, что

$$
N=2^{\log _{2}(N+1)}-1 \leqslant 2^{\left\lceil\log _{2}(N+1)\right\rceil}-1=2^{m}-1 .
$$


Лемма 4. Набор $\left(S_{1}^{\prime}, \ldots, S_{m}^{\prime}\right)$ является проверяющим тестом.

Доказательство. Пусть $H_{1}$ и $H_{2}$ - две произвольные неисправности системы элементов, при которых множества неисправных элементов различны. Надо доказать, что наборы функций, реализуемых схемами $S_{1}^{\prime}, \ldots, S_{m}^{\prime}$ при этих двух неисправностях, не совпадают. Предположим сначала, что при одной из неисправностей $H_{1}$ и $H_{2}$ (без ограничения общности, при $H_{1}$ ) все элементы исправны. Тогда при неисправности $H_{2}$ некоторый элемент $E_{i}$ неисправен. Так как $1 \leqslant i \leqslant N \leqslant 2^{m}-1$ в силу (6), то существует такое $j \in\{1, \ldots, m\}$, что в $j$-м справа разряде двоичной записи числа $i$ стоит единица. Это означает, что $E_{i} \in M_{j}$ и, следовательно, элемент $E_{i}$ содержится в схеме $S_{j}^{\prime}$. Тогда в силу леммы 3 схема $S_{j}^{\prime}$ реализует функцию $x$ при неисправности $H_{1}$ и некоторую булеву константу при неисправности $H_{2}$, откуда следует, что наборы функций, реализуемых схемами $S_{1}^{\prime}, \ldots, S_{m}^{\prime}$ при этих двух неисправностях, не совпадают.

Пусть теперь при неисправности $H_{1}$ неисправен некоторый элемент $E_{i_{1}}$, а при неисправности $H_{2}-$ некоторый элемент $E_{i_{2}}$. Тогда $i_{1} \neq i_{2}$. Так как $i_{1} \leqslant N \leqslant 2^{m}-1$ и $i_{2} \leqslant N \leqslant 2^{m}-1$ в силу (6), то существует такое $j \in\{1, \ldots, m\}$, что в $j$-м справа разряде двоичной записи одного из чисел $i_{1}, i_{2}$ (без ограничения общности, $i_{1}$ ) стоит единица, а другого $\left(i_{2}\right)$ - нуль. Это означает, что $E_{i_{1}} \in M_{j}$ и $E_{i_{2}} \notin M_{j}$, т. е. в схеме $S_{j}^{\prime}$ содержится элемент $E_{i_{1}}$ и не содержится элемента $E_{i_{2}}$. Тогда в силу леммы 3 схема $S_{j}^{\prime}$ реализует некоторую булеву константу при неисправности $H_{1}$ и функцию $x$ при неисправности $H_{2}$, откуда следует, что наборы функций, реализуемых схемами $S_{1}^{\prime}, \ldots, S_{m}^{\prime}$ при этих двух неисправностях, не совпадают. Лемма 4 доказана.

Из леммы 4 получаем соотношение $L(f, N) \leqslant m=\left\lceil\log _{2}(N+1)\right\rceil$. Случай 3 разобран.

Осталось получить оценку $L(f, N) \leqslant\left\lceil\log _{3}(2 N+1)\right\rceil$ в случае 4. Докажем предварительно две общие леммы, которые могут использоваться отдельно от доказываемой в данной работе теоремы. Для того, чтобы подчеркнуть этот факт, будем использовать в этих леммах независимую систему обозначений, которые частично могут пересекаться с обозначениями, используемыми при постановке задачи и доказательстве основной теоремы.

Лемма 5. Пусть $N$ - натуральное число, $r=\left\lceil\log _{3}(2 N+1)\right\rceil$. Тогда существуют такие целье неотрицательные числа $n_{i, j}, i=1, \ldots, r, j=1, \ldots, C_{r}^{i}$, что $\sum_{i=1}^{r} \sum_{j=1}^{C_{r}^{i}} n_{i, j}=N$, причём каждое $n_{i, j}$ не превосходит $2^{i-1}$ u $n_{1,1}=n_{1,2}=\ldots=$ $n_{1, r}=1$.

Доказательство. Из определения числа $r$ легко видеть, что всегда $N \geqslant r$ и $3^{r} \geqslant$ $2 N+1$, откуда

$$
r \leqslant N \leqslant \frac{3^{r}-1}{2}
$$

Далее, пусть

$$
n_{i, j}^{\prime}=2^{i-1}, i=1, \ldots, r, j=1, \ldots, C_{r}^{i} .
$$

Тогда $n_{1,1}^{\prime}=n_{1,2}^{\prime}=\ldots=n_{1, r}^{\prime}=1$ и

$$
\sum_{i=1}^{r} \sum_{j=1}^{C_{r}^{i}} n_{i, j}^{\prime}=\sum_{i=1}^{r} \sum_{j=1}^{C_{r}^{i}} 2^{i-1}=\sum_{i=1}^{r} C_{r}^{i} 2^{i-1}=\frac{1}{2}\left(\sum_{i=0}^{r} C_{r}^{i} 2^{i}-1\right)=\frac{1}{2}\left((1+2)^{r}-1\right)=\frac{3^{r}-1}{2},
$$


откуда

$$
\sum_{i=2}^{r} \sum_{j=1}^{C_{r}^{i}} n_{i, j}^{\prime}=\frac{3^{r}-1}{2}-\sum_{j=1}^{C_{r}^{1}} n_{1, j}^{\prime}=\frac{3^{r}-1}{2}-\sum_{j=1}^{r} 1=\frac{3^{r}-1}{2}-r .
$$

Из последнего соотношения и (7) получаем неравенства

$$
\sum_{i=2}^{r} \sum_{j=1}^{C_{r}^{i}} n_{i, j}^{\prime} \geqslant N-r \geqslant 0
$$

Из (9) нетрудно заключить, что существуют такие целые неотрицательные числа $n_{i, j}, i=2, \ldots, r, j=1, \ldots, C_{r}^{i}$, что

$$
n_{i, j} \leqslant n_{i, j}^{\prime}
$$

И

$$
\sum_{i=2}^{r} \sum_{j=1}^{C_{r}^{i}} n_{i, j}=N-r
$$

(действительно, последовательно уменьшая на единицу те из чисел $n_{i, j}^{\prime}$, которые больше нуля, можно добиться того, чтобы сумма этих чисел стала равна $N-r)$. Пусть $n_{1,1}=n_{1,2}=\ldots=n_{1, r}=1$. Тогда отсюда и из (8), (10) следует, что $n_{i, j} \leqslant$ $2^{i-1}, i=1, \ldots, r, j=1, \ldots, C_{r}^{i}$, а из $(11)$ - что

$$
\sum_{i=1}^{r} \sum_{j=1}^{C_{r}^{i}} n_{i, j}=\sum_{j=1}^{C_{r}^{1}} n_{1, j}+\sum_{i=2}^{r} \sum_{j=1}^{C_{r}^{i}} n_{i, j}=\sum_{j=1}^{r} 1+N-r=N .
$$

Лемма 5 доказана.

Пусть $h-$ произвольное натуральное число, $A_{1}, \ldots, A_{h}$ - произвольные множества. Через $A_{1} \sqcup \ldots \sqcup A_{h}$ будем обозначать объединение множеств $A_{1}, \ldots, A_{h}$ при условии, что эти множества попарно не пересекаются. Таким образом, значок $\sqcup$ будет иметь смысл обычного объединения множеств, дополнительно указывая, что множества, между которыми стоит один или несколько таких значков, попарно не пересекаются. Будем использовать также обозначение $\bigsqcup_{i=1}^{h} A_{i}=A_{1} \sqcup \ldots \sqcup A_{h}$.

Лемма 6. Пусть $A-$ конечное множество, $|A|=n \geqslant 1, s=\left\lceil\log _{2} n\right\rceil+1$. Тогда существуют $2 s$ множеств $A_{1,1}, A_{1,2}, A_{2,1}, A_{2,2}, \ldots, A_{s, 1}, A_{s, 2}$, обладающих следующими свойствами:

1) для любого $i \in\{1, \ldots, s-1\}$ выполнено неравенство ||$A_{i, 1}|-| A_{i, 2}|| \leqslant 1$;

2) ||$A_{s, 1}|-| A_{s, 2}|| \leqslant 2$;

3) для любого $i \in\{1, \ldots, s\}$ выполнено соотношение $A=A_{i, 1} \sqcup A_{i, 2}$;

4) для любого двухэлементного подмножества $B$ множества $A$ существуют такие индексы $i_{1}, i_{2} \in\{1, \ldots, s\}$, что $B \subseteq A_{i_{1}, 1}$ или $B \subseteq A_{i_{1}, 2}$ и при этом $B \nsubseteq A_{i_{2}, 1}$, $B \nsubseteq A_{i_{2}, 2}$.

Замечание 3. Лемма 6 допускает переформулировку на языке теории графов. А именно, пусть $D_{n}$ - полный граф на $n$ вершинах, где $n \geqslant 1 ; s=\left\lceil\log _{2} n\right\rceil+1$. Тогда существуют такие $s$ раскрасок вершин графа $D_{n}$ в чёрный и белый цвета, что при 
каждой из них, кроме $s$-й, число чёрных вершин отличается от числа белых вершин не более чем на 1 , а при $s$-й - не более чем на 2 и при этом любое ребро графа $D_{n}$ хотя бы при одной раскраске имеет одноцветные вершины и хотя бы при одной раскраске имеет разноцветные вершины.

Доказательство леммы 6. Пусть $A=\left\{a_{1}, \ldots, a_{n}\right\}$. (Напомним, что в лемме 6 используется независимая система обозначений; в частности, число $n$ не имеет отношения к числу $n$ в постановке задачи как к числу переменных у функции $f$.) Определим множество $A_{s, 1}$ как множество всех элементов из $A$, номера которых имеют вид $2 k-1$ или $2\left\lfloor\frac{n}{2}\right\rfloor-2 k+2$, где $k=1, \ldots,\left\lceil\frac{\lfloor n / 2\rfloor}{2}\right]$. (В случае $n=1$ множество $A_{s, 1}$ окажется пустым.) Так как $2\left\lfloor\frac{n}{2}\right\rfloor-2 k+2 \leqslant 2\left\lfloor\frac{n}{2}\right\rfloor \leqslant n$ при $k \geqslant 1$ и для любых $k_{1}, k_{2} \in\left\{1, \ldots,\left\lceil\frac{\lfloor n / 2\rfloor}{2}\right\rceil\right\}$ выполняются соотношения

$$
2 k_{1}-1 \leqslant 2\left\lceil\frac{\lfloor n / 2\rfloor}{2}\right\rceil-1 \leqslant 2 \cdot \frac{\lfloor n / 2\rfloor+1}{2}-1=\left\lfloor\frac{n}{2}\right\rfloor,
$$

аналогично

$$
2 k_{2}-1 \leqslant\left\lfloor\frac{n}{2}\right\rfloor
$$

и, как следствие,

$$
2\left\lfloor\frac{n}{2}\right\rfloor-2 k_{2}+2=2\left\lfloor\frac{n}{2}\right\rfloor+1-\left(2 k_{2}-1\right) \geqslant 2\left\lfloor\frac{n}{2}\right\rfloor+1-\left\lfloor\frac{n}{2}\right\rfloor=\left\lfloor\frac{n}{2}\right\rfloor+1>2 k_{1}-1,
$$

то $\left|A_{s, 1}\right|=2\left\lceil\frac{\lfloor n / 2\rfloor}{2}\right\rceil$.

Пусть $A_{s, 2}=A \backslash A_{s, 1}$. Тогда $A=A_{s, 1} \sqcup A_{s, 2}$ и

$$
|| A_{s, 1}|-| A_{s, 2}||=|| A_{s, 1}|-| A|+| A_{s, 1}||=\left|4\left\lceil\frac{\lfloor n / 2\rfloor}{2}\right\rceil-n\right| \leqslant 2,
$$

так каK

$$
4\left\lceil\frac{\lfloor n / 2\rfloor}{2}\right\rceil \leqslant 4 \cdot \frac{\lfloor n / 2\rfloor+1}{2}=2\left\lfloor\frac{n}{2}\right\rfloor+2 \leqslant n+2
$$

И

$$
4\left\lceil\frac{\lfloor n / 2\rfloor}{2}\right\rceil \geqslant 4 \cdot \frac{\lfloor n / 2\rfloor}{2}=2\left\lfloor\frac{n}{2}\right\rfloor \geqslant 2 \cdot \frac{n-1}{2}=n-1 .
$$

(Отметим, кстати, что равенство ||$A_{s, 1}|-| A_{s, 2}||=2$ может достигаться только при обращении обоих неравенств в (12) в равенства, т. е. только при $\left\lceil\frac{\lfloor n / 2\rfloor}{2}\right\rceil=$ $\frac{\lfloor n / 2\rfloor+1}{2}$ и $2\left\lfloor\frac{n}{2}\right\rfloor=n$, а это означает, что число $n$ должно быть представимо в виде $4 k-2$, где $k$ - натуральное число. Для всех остальных $n$ справедливо неравенство ||$A_{s, 1}|-| A_{s, 2}|| \leqslant 1$.)

Далее, в случае $s \geqslant 2$ определим множество $A_{s-1,1}$ как множество всех элементов из $A$, номера которых имеют вид $2 k-1$, где $k=1, \ldots,\left\lfloor\frac{n}{2}\right\rfloor$. Тогда $\left|A_{s-1,1}\right|=\left\lfloor\frac{n}{2}\right\rfloor$. Пусть $A_{s-1,2}=A \backslash A_{s-1,1}$. Тогда $A=A_{s-1,1} \sqcup A_{s-1,2}$ и

$$
|| A_{s-1,1}|-| A_{s-1,2}||=|| A_{s-1,1}|-| A|+| A_{s-1,1}||=\left|2\left\lfloor\frac{n}{2}\right\rfloor-n\right| \leqslant 1 .
$$

В случае $s \geqslant 3$ определим по индукции целые числа $m_{i, j}$ для $i=1, \ldots, s-2$, $j=1, \ldots, 2^{i}$. Положим

$$
m_{1,1}=m_{1,2}=\left\lfloor\frac{n}{2}\right\rfloor .
$$


Пусть уже определены целые числа $m_{i-1, j}, i \in\{2, \ldots, s-2\}, j=1, \ldots, 2^{i-1}$. Для каждого $j \in\left\{1, \ldots, 2^{i-2}\right\}$ положим

$$
\begin{gathered}
m_{i, 2 j-1}=m_{i, 2^{i}-2 j+2}=\left\lfloor\frac{m_{i-1, j}}{2}\right\rfloor, \\
m_{i, 2 j}=m_{i, 2^{i}-2 j+1}=\left\lceil\frac{m_{i-1, j}}{2}\right\rceil .
\end{gathered}
$$

Легко видеть, что таким образом определены целые числа $m_{i, j}$ для каждых $i=$ $1, \ldots, s-2$ и $j=1, \ldots, 2^{i}$, причём для любых $i \in\{1, \ldots, s-2\}, j \in\left\{1, \ldots, 2^{i}\right\}$ выполняется соотношение

$$
m_{i, j}=m_{i, 2^{i}-j+1} \text {. }
$$

Кроме того, для любых $i \in\{2, \ldots, s-2\}, j \in\left\{1, \ldots, 2^{i-1}\right\}$ справедливо равенство

$$
m_{i, 2 j-1}+m_{i, 2 j}=m_{i-1, j} .
$$

Действительно, если $j \in\left\{1, \ldots, 2^{i-2}\right\}$, то это верно в силу (14), (15), а если $j \in$ $\left\{2^{i-2}+1, \ldots, 2^{i-1}\right\}$, то, вводя обозначение $j^{\prime}=2^{i-1}-j+1$, получим, что $j^{\prime} \in$ $\left\{1, \ldots, 2^{i-2}\right\}$ и

$$
\begin{aligned}
& m_{i, 2 j-1}+m_{i, 2 j}=m_{i, 2^{i}-2 j+2}+m_{i, 2^{i}-2 j+1}=m_{i, 2 j^{\prime}}+m_{i, 2 j^{\prime}-1}= \\
& \quad=m_{i-1, j^{\prime}}=m_{i-1,2^{i-1}-j+1}=m_{i-1, j}
\end{aligned}
$$

в силу (14)-(16).

Отметим также, что для любого $i \in\{1, \ldots, s-2\}$ выполняется равенство

$$
\sum_{j=1}^{2^{i}} m_{i, j}=2\left\lfloor\frac{n}{2}\right\rfloor \text {. }
$$

В самом деле, для $i=1$ это верно в силу (13); если (18) выполняется для $i=t-1 \in$ $\{1, \ldots, s-3\}$, то

$$
\sum_{j=1}^{2^{t}} m_{t, j}=\sum_{j=1}^{2^{t-1}}\left(m_{t, 2 j-1}+m_{t, 2 j}\right)=\sum_{j=1}^{2^{t-1}} m_{t-1, j}=2\left\lfloor\frac{n}{2}\right\rfloor
$$

в силу (17), и поэтому (18) справедливо и для $i=t$.

Для каждых $i \in\{1, \ldots, s-2\}$ и $j \in\left\{1, \ldots, 2^{i}\right\}$ определим множество $B_{i, j}$ как множество всех элементов из $A$, номера которых принадлежат отрезку $\left[\sum_{t=1}^{j-1} m_{i, t}+1 ; \sum_{t=1}^{j} m_{i, t}\right]$. (Здесь и далее будем полагать, что $\sum_{t=1}^{0} m_{i, t}=0$.) Например, $B_{i, 1}=\left\{a_{1}, \ldots, a_{m_{i, 1}}\right\}, B_{i, 2}=\left\{a_{m_{i, 1}+1}, \ldots, a_{m_{i, 1}+m_{i, 2}}\right\}$ и т.д. Ясно, что множества $B_{i, j}$ попарно не пересекаются при различных $j$ (при фиксированном $i$ ). Из (18) следует, что $\sum_{t=1}^{2^{i}} m_{i, t}=2\left\lfloor\frac{n}{2}\right\rfloor \leqslant n=|A|$, откуда заключаем, что

$$
\left|B_{i, j}\right|=m_{i, j}
$$

и

$$
\bigsqcup_{j=1}^{2^{i}} B_{i, j}=\left\{a_{1}, \ldots, a_{2\left\lfloor\frac{n}{2}\right\rfloor}\right\}
$$


Пусть

$$
A_{i, 2}=\bigsqcup_{j=1}^{2^{i-1}} B_{i, 2 j}
$$

для $i=1, \ldots, s-2$. Тогда в силу $(16),(18),(19)$ имеем

$$
\begin{aligned}
\left|A_{i, 2}\right|=\sum_{j=1}^{2^{i-1}}\left|B_{i, 2 j}\right|=\sum_{j=1}^{2^{i-1}} m_{i, 2 j} & =\frac{1}{2}\left(\sum_{j=1}^{2^{i-1}} m_{i, 2 j}+\sum_{j=1}^{2^{i-1}} m_{i, 2^{i}-2 j+1}\right) \\
& =\frac{1}{2}\left(\sum_{j=1}^{2^{i-1}} m_{i, 2 j}+\sum_{j^{\prime}=1}^{2^{i-1}} m_{i, 2 j^{\prime}-1}\right)=\frac{1}{2} \sum_{j=1}^{2^{i}} m_{i, j}=\left\lfloor\frac{n}{2}\right\rfloor .
\end{aligned}
$$

Пусть $A_{i, 1}=A \backslash A_{i, 2}$ для $i=1, \ldots, s-2$. Тогда $A=A_{i, 1} \sqcup A_{i, 2}$ и

$$
|| A_{i, 1}|-| A_{i, 2}||=|A-| A_{i, 2}|-| A_{i, 2}||=\left|n-2\left\lfloor\frac{n}{2}\right\rfloor\right| \leqslant 1 .
$$

Из (20) вытекает, что

$$
A_{i, 1}= \begin{cases}\bigsqcup_{j=1}^{2-1} B_{i, 2 j-1}, & \text { если } n \text { чётно, } \\ \bigsqcup_{j=1}^{i-1} B_{i, 2 j-1} \sqcup\left\{a_{n}\right\}, & \text { если } n \text { нечётно. }\end{cases}
$$

Таким образом, построены $2 s$ множеств $A_{1,1}, A_{1,2}, A_{2,1}, A_{2,2}, \ldots, A_{s, 1}, A_{s, 2}$, для которых выполнены свойства 1)-3) из условия леммы 6.

Пример 1. Пусть $n=23$, тогда $s=\left\lceil\log _{2} 23\right\rceil+1=6, m_{1,1}=m_{1,2}=11$, $m_{2,1}=m_{2,4}=5, m_{2,2}=m_{2,3}=6, m_{3,1}=m_{3,8}=2, m_{3,2}=m_{3,3}=m_{3,4}=m_{3,5}=$ $m_{3,6}=m_{3,7}=3, m_{4,1}=m_{4,2}=m_{4,3}=m_{4,5}=m_{4,7}=m_{4,10}=m_{4,12}=m_{4,14}=$ $m_{4,15}=m_{4,16}=1, m_{4,4}=m_{4,6}=m_{4,8}=m_{4,9}=m_{4,11}=m_{4,13}=2$ и множества $A_{1,1}, A_{1,2}, \ldots, A_{6,1}, A_{6,2}$ имеют следующий вид:

$$
\begin{array}{ll}
A_{1,1}=\{1,2,3,4,5,6,7,8,9,10,11,23\}, & A_{1,2}=\{12,13,14,15,16,17,18,19,20,21,22\}, \\
A_{2,1}=\{1,2,3,4,5,12,13,14,15,16,17,23\}, & A_{2,2}=\{6,7,8,9,10,11,18,19,20,21,22\}, \\
A_{3,1}=\{1,2,6,7,8,12,13,14,18,19,20,23\}, & A_{3,2}=\{3,4,5,9,10,11,15,16,17,21,22\}, \\
A_{4,1}=\{1,3,6,9,12,13,15,16,18,19,21,23\}, & A_{4,2}=\{2,4,5,7,8,10,11,14,17,20,22\}, \\
A_{5,1}=\{1,3,5,7,9,11,13,15,17,19,21\}, & A_{5,2}=\{2,4,6,8,10,12,14,16,18,20,22,23\}, \\
A_{6,1}=\{1,3,5,7,9,11,12,14,16,18,20,22\}, & A_{6,2}=\{2,4,6,8,10,13,15,17,19,21,23\} .
\end{array}
$$

(Для удобства элемент с номером $j$ обозначаем просто $j$, где $j=1, \ldots, 23$. )

Графически это можно изобразить так, как показано на рис. 2. Если на пересечении $i$-й строки и $j$-го столбца стоит чёрный кружок, то элемент $a_{j}$ принадлежит множеству $A_{i, 1}$; в противном случае данный элемент принадлежит множеству $A_{i, 2}$.

Осталось доказать выполнение свойства 4) из условия леммы 6 для построенных множеств $A_{1,1}, A_{1,2}, \ldots, A_{s, 1}, A_{s, 2}$. В случае $n=1$ оно тривиально (у $A$ нет ни одного двухэлементного подмножества). В случае $n=2$ по построению имеем $s=\left\lceil\log _{2} n\right\rceil+1=2, A_{1,1}=\left\{a_{1}\right\}, A_{1,2}=\left\{a_{2}\right\}, A_{2,1}=A, A_{2,2}=\varnothing ;$ в то же время, единственным двухэлементным подмножеством множества $A$ является само $A$, 


\begin{tabular}{|c|c|c|c|c|c|c|c|c|c|c|c|c|c|c|c|c|c|c|c|c|c|c|c|}
\hline $\begin{array}{l}\text { элемент } \\
\text { множества }\end{array}$ & 1 & 2 & 3 & 4 & 5 & 6 & 7 & 8 & 9 & 10 & 11 & 12 & 13 & 14 & 15 & 16 & 17 & 18 & 19 & 20 & 212 & 222 & 23 \\
\hline$A_{1,1}$ и $A_{1,2}$ & & & & & & & & & & & 0 & & & & & & & & & & & & \\
\hline$A_{2,1} \backsim A_{2,2}$ & & & & & & & & & & & & & & & & & & & & & & & \\
\hline$A_{3,1} \backsim A_{3,2}$ & & & & & & & & & & & & & & 0 & & & & & & O & & & \\
\hline$A_{4,1} \backsim A_{4,2}$ & 0 & & 0 & & & 0 & & & O & & & O & 0 & & 0 & 0 & & 0 & 10 & & 0 & & \\
\hline$A_{5,1}$ и $A_{5,2}$ & 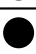 & & P & & 0 & & 0 & & O & & 0 & & 0 & & 0 & & O & & 0 & & 0 & & \\
\hline$A_{6,1}$ и $A_{6,2}$ & 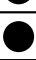 & & 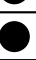 & & 0 & & 0 & & 0 & & & 0 & & 0 & & 0 & & & & 0 & & 0 & \\
\hline
\end{tabular}

Рис. 2

поэтому при $i_{1}=2, i_{2}=1$ свойство 4) выполняется. Пусть теперь $n \geqslant 3$. Тогда $s=\left\lceil\log _{2} n\right\rceil+1 \geqslant 3$. Из (17) следует, что для любых $i \in\{2, \ldots, s-2\}, j \in\left\{1, \ldots, 2^{i-1}\right\}$ справедливы равенства

$$
\begin{aligned}
\sum_{t=1}^{j-1} m_{i-1, t}+1 & =\sum_{t=1}^{2 j-2} m_{i, t}+1, \\
\sum_{t=1}^{j} m_{i-1, t} & =\sum_{t=1}^{2 j} m_{i, t},
\end{aligned}
$$

откуда получаем, что любое целое число из отрезка $\left[\sum_{t=1}^{j-1} m_{i-1, t}+1 ; \sum_{t=1}^{j} m_{i-1, t}\right]$ содержится ровно в одном из отрезков $\left[\sum_{t=1}^{2 j-2} m_{i, t}+1 ; \sum_{t=1}^{2 j-1} m_{i, t}\right],\left[\sum_{t=1}^{2 j-1} m_{i, t}+1 ; \sum_{t=1}^{2 j} m_{i, t}\right]$. Согласно определению множеств $B_{i, j}$ это означает, что $B_{i-1, j}=B_{i, 2 j-1} \sqcup B_{i, 2 j}$ для любых $i \in\{2, \ldots, s-2\}, j \in\left\{1, \ldots, 2^{i-1}\right\}$. Отсюда и из (20)-(22) следует, что для любых $i \in\{2, \ldots, s-2\}, j \in\left\{1, \ldots, 2^{i-1}\right\}$ выполняются соотношения

$$
\begin{gathered}
B_{i-1, j} \cap A_{i, 1}=B_{i, 2 j-1}, \\
B_{i-1, j} \cap A_{i, 2}=B_{i, 2 j},
\end{gathered}
$$

которые можно объединить в одно, записав

$$
B_{i-1, j} \cap A_{i, b}=B_{i, 2 j+b-2},
$$

где $b \in\{1 ; 2\}$.

Далее, для любых $i \in\{1, \ldots, s-2\}, j \in\left\{1, \ldots, 2^{i}\right\}$ выполняются неравенства

$$
2^{s-2-i} \leqslant m_{i, j} \leqslant 2^{s-1-i} \text {. }
$$

Действительно, из равенства $s=\left\lceil\log _{2} n\right\rceil+1$ следует соотношение $2^{s-2} \leqslant n \leqslant 2^{s-1}$, а отсюда и из (13) вытекают неравенства (24) в случае $i=1$; если же соотношение (24) выполнено для $i=t-1 \in\{1, \ldots, s-3\}$, то из (14), (15) следует его справедливость и для $i=t$.

Предположим, что существует такое двухэлементное подмножество $B$ множества $A$, что для любого индекса $i_{2} \in\{1, \ldots, s\}$ либо $B \subseteq A_{i_{2}, 1}$, либо $B \subseteq A_{i_{2}, 2}$. Будем считать, что $B \subseteq A_{i_{2}, b_{i_{2}}}$, где $b_{i_{2}} \in\{1 ; 2\}$. Рассмотрим 2 случая.

1. Пусть $n$ чётно или $a_{n} \notin B$. Тогда при $i_{2}=1$ в силу $(21),(22)$ и соотношения $B \subseteq A_{1, b_{1}}$ имеем $B \subseteq B_{1, b_{1}}$. Определим натуральные числа $d_{1}, \ldots, d_{s-2}$ (напомним, 
что $s \geqslant 3)$. Пусть $d_{1}=b_{1}$, тогда $1 \leqslant d_{1} \leqslant 2=2^{1}$ и $B \subseteq B_{1, d_{1}}$. Если $s \geqslant 4$, то при $i_{2}=2$ в силу предположения имеем $B \subseteq A_{2, b_{2}}$, а в таком случае $B \subseteq B_{1, d_{1}} \cap A_{2, b_{2}}=$ $B_{2,2 d_{1}+b_{2}-2}$ в силу (23). Пусть $d_{2}=2 d_{1}+b_{2}-2$, тогда $1 \leqslant d_{2} \leqslant 4=2^{2}$ и $B \subseteq B_{2, d_{2}}$. Далее, если $s \geqslant 5$, то при $i_{2}=3$ в силу предположения имеем $B \subseteq A_{3, b_{3}}$, а в таком случае $B \subseteq B_{2, d_{2}} \cap A_{3, b_{3}}=B_{3,2 d_{2}+b_{3}-2}$ в силу (23), и после введения обозначения $d_{3}=2 d_{2}+b_{3}-2$ получаем $1 \leqslant d_{3} \leqslant 8=2^{3}$ и $B \subseteq B_{3, d_{3}}$. Рассуждая аналогично для $i_{2}=4,5, \ldots, s-2$, получим, что $B \subseteq B_{s-2, d_{s-2}}$, где $1 \leqslant d_{s-2} \leqslant 2^{s-2}$. Из (19) и (24) заключаем, что $\left|B_{s-2, d_{s-2}}\right|=m_{s-2, d_{s-2}} \leqslant 2$. Отсюда, из соотношений $|B|=2$, $B \subseteq B_{s-2, d_{s-2}},(20)$ и определения множеств $B_{i, j}$ следует, что $B=B_{s-2, d_{s-2}}=$ $\left\{a_{l}, a_{l+1}\right\}$, где $2 \leqslant l+1 \leqslant 2\left\lfloor\frac{n}{2}\right\rfloor$. Далее, по предположению $B \subseteq A_{s-1, b_{s-1}}$, где $b_{s-1} \in\{1 ; 2\}$. Из определения множеств $A_{s-1,1}$ и $A_{s-1,2}$ легко видеть, что среди элементов с номерами от 1 до $2\left\lfloor\frac{n}{2}\right\rfloor$ во множестве $A_{s-1,1}$ содержатся в точности все элементы с нечётными, а в множестве $A_{s-2,2}-$ с чётными номерами. Но номера $l$ и $l+1$ имеют разную чётность и принадлежат отрезку $\left[1 ; 2\left\lfloor\frac{n}{2}\right\rfloor\right]$, откуда следует, что $B=\left\{a_{l}, a_{l+1}\right\} \nsubseteq A_{s-1, b_{s-1}}$. Получено противоречие.

2. Пусть $n$ нечётно и $a_{n} \in B$. В этом случае из (22) следует, что $a_{n} \in A_{i_{2}, 1}$ для любого $i_{2} \in\{1, \ldots, s-2\}$, а значит, $a_{n} \notin A_{i_{2}, 2}$. В таком случае, так как по предположению $B \subseteq A_{i_{2}, b_{i_{2}}}$, где $b_{i_{2}} \in\{1 ; 2\}$, то $b_{i_{2}}=1$ и $B \subseteq A_{i_{2}, 1}$. Пусть $a_{l}-$ элемент, содержащийся во множестве $B$ и отличный от элемента $a_{n}$. Тогда $a_{l} \in A_{i_{2}, 1}$ для любого $i_{2} \in\{1, \ldots, s-2\}$, причём $l<n$. В таком случае при $i_{2}=1$ в силу $(22)$ имеем $a_{l} \in A_{1,1} \backslash\left\{a_{n}\right\}=B_{1,1}$. Если $s \geqslant 4$, то при $i_{2}=2$ имеем $a_{l} \in A_{2,1}$, а тогда $a_{l} \in B_{1,1} \cap A_{2,1}=B_{2,2 \cdot 1+1-2}=B_{2,1}$ в силу (23). Далее, если $s \geqslant 5$, то при $i_{2}=3$ имеем $a_{l} \in A_{3,1}$, а в таком случае $a_{l} \in B_{2,1} \cap A_{3,1}=B_{3,2 \cdot 1+1-2}=B_{3,1}$ в силу (23). Рассуждая аналогично для $i_{2}=4,5, \ldots, s-2$, получим, что $a_{l} \in B_{s-2,1}$. Из равенства $s=\left\lceil\log _{2} n\right\rceil+1$ следуют неравенства $2^{s-2} \leqslant n \leqslant 2^{s-1}$, а отсюда и из нечётности $n$ - неравенства $2^{s-2}<n \leqslant 2^{s-1}-1$. Из (13), (14) и последнего соотношения следует, что $2^{s-3} \leqslant m_{1,1} \leqslant 2^{s-2}-1,2^{s-4} \leqslant m_{2,1}=\left\lfloor\frac{m_{1,1}}{2}\right\rfloor \leqslant 2^{s-3}-1$ (в случае $s \geqslant 4$ ), .., $2^{0} \leqslant m_{s-2,1} \leqslant 2^{1}-1$, т. е. $m_{s-2,1}=1$, а тогда по определению множества $B_{s-2,1}$ оно равно $\left\{a_{1}\right\}$. Получаем, что $l=1$ и $B=\left\{a_{1}, a_{n}\right\}$. Из определения множеств $A_{s-1,1}$ и $A_{s-1,2}$ видно, что $a_{n} \notin A_{s-1,1}, a_{1} \notin A_{s-1,2}$, откуда следует, что $B \nsubseteq A_{s-1,1}$ и $B \nsubseteq$ $A_{s-1,2}$. Но это противоречит тому, что $B \subseteq A_{s-1, b_{s-1}}$ для некоторого $b_{s-1} \in\{1 ; 2\}$.

Таким образом, предположение о существовании такого двухэлементного подмножества $B$ множества $A$, что для любого индекса $i_{2} \in\{1, \ldots, s\}$ либо $B \subseteq A_{i_{2}, 1}$, либо $B \subseteq A_{i_{2}, 2}$, неизбежно приводит к противоречию. Значит, для любого двухэлементного подмножества $B$ множества $A$ существует такой индекс $i_{2} \in\{1, \ldots, s\}$, что $B \nsubseteq A_{i_{2}, 1}$ и $B \nsubseteq A_{i_{2}, 2}$. Осталось доказать, что для любого двухэлементного подмножества $B$ множества $A$ существует такой индекс $i_{1} \in\{1, \ldots, s\}$, что $B \subseteq A_{i_{1}, 1}$ или $B \subseteq A_{i_{1}, 2}$. Предположим, что это не так, т. е. существует такое подмножество $B=\left\{a_{l}, a_{m}\right\}$ множества $A$, что для любого индекса $i_{1} \in\{1, \ldots, s\}$ одновременно $B \nsubseteq A_{i_{1}, 1}$ и $B \nsubseteq A_{i_{1}, 2}$. Из этого предположения с учётом того, что $A_{i_{1}, 1} \sqcup A_{i_{1}, 2}=A$, следует, что $a_{l} \in A_{i_{1}, b_{i_{1}}}$ и $a_{m} \in A_{i_{1}, 3-b_{i_{1}}}$ для некоторого $b_{i_{1}} \in\{1 ; 2\}$. Рассмотрим 2 случая.

1. Пусть $n$ чётно или $a_{n} \notin B$. Тогда при $i_{1}=1$ в силу $(21),(22)$ и соотношений $a_{l} \in A_{1, b_{1}}, a_{m} \in A_{1,3-b_{1}}$ имеем $a_{l} \in B_{1, b_{1}}, a_{m} \in B_{1,3-b_{1}}$. Определим натуральные числа $d_{1}, d_{1}^{\prime}, \ldots, d_{s-2}, d_{s-2}^{\prime}$. Пусть $d_{1}=b_{1}, d_{1}^{\prime}=3-d_{1}$, тогда $d_{1}+d_{1}^{\prime}=3=2^{1}+1$, $1 \leqslant d_{1} \leqslant 2=2^{1}, 1 \leqslant d_{1}^{\prime}=2^{1}+1-d_{1} \leqslant 2^{1}, a_{l} \in B_{1, d_{1}}$ и $a_{m} \in B_{1, d_{1}^{\prime}}$. Если $s \geqslant 4$, то при $i_{1}=2$ в силу предположения имеем $a_{l} \in A_{2, b_{2}}, a_{m} \in A_{2,3-b_{2}}$, а в таком случае $a_{l} \in B_{1, d_{1}} \cap A_{2, b_{2}}=B_{2,2 d_{1}+b_{2}-2}$ и $a_{m} \in B_{1, d_{1}^{\prime}} \cap A_{2,3-b_{2}}=B_{2,2 d_{1}^{\prime}+\left(3-b_{2}\right)-2}$ в силу (23). 
Пусть $d_{2}=2 d_{1}+b_{2}-2, d_{2}^{\prime}=2 d_{1}^{\prime}+\left(3-b_{2}\right)-2$, тогда $d_{2}+d_{2}^{\prime}=2\left(d_{1}+d_{1}^{\prime}\right)-1=2^{2}+1$, $1 \leqslant d_{2} \leqslant 4=2^{2}, 1 \leqslant d_{2}^{\prime}=2^{2}+1-d_{2} \leqslant 2^{2}, a_{l} \in B_{2, d_{2}}$ и $a_{m} \in B_{2, d_{2}^{\prime}}$. Далее, если $s \geqslant 5$, то при $i_{1}=3$ в силу предположения имеем $a_{l} \in A_{3, b_{3}}, a_{m} \in A_{3,3-b_{3}}$, а в таком случае $a_{l} \in B_{2, d_{2}} \cap A_{3, b_{3}}=B_{2,2 d_{2}+b_{3}-2}$ и $a_{m} \in B_{2, d_{2}^{\prime}} \cap A_{3,3-b_{3}}=B_{2,2 d_{2}^{\prime}+\left(3-b_{3}\right)-2}$ в силу (23), и после введения обозначений $d_{3}=2 d_{2}+b_{3}-2, d_{3}^{\prime}=2 d_{2}^{\prime}+\left(3-b_{3}\right)-2$ получаем $d_{3}+d_{3}^{\prime}=2\left(d_{2}+d_{2}^{\prime}\right)-1=2^{3}+1,1 \leqslant d_{3} \leqslant 8=2^{3}, 1 \leqslant d_{3}^{\prime}=2^{3}+1-d_{3} \leqslant 2^{3}$, $a_{l} \in B_{3, d_{3}}$ и $a_{m} \in B_{3, d_{3}^{\prime}}$. Рассуждая аналогично для $i_{1}=4,5, \ldots, s-2$, получим, что $a_{l} \in B_{s-2, d_{s-2}}$ и $a_{m} \in B_{s-2, d_{s-2}^{\prime}}$, где

$$
d_{s-2}+d_{s-2}^{\prime}=2^{s-2}+1
$$

$1 \leqslant d_{s-2} \leqslant 2^{s-2}$ и $1 \leqslant d_{s-2}^{\prime} \leqslant 2^{s-2}$. Из (16), (19), (24) и (25) заключаем, что $\left|B_{s-2, d_{s-2}}\right|=m_{s-2, d_{s-2}} \leqslant 2,\left|B_{s-2, d_{s-2}^{\prime}}\right|=m_{s-2, d_{s-2}^{\prime}}=m_{s-2,2^{s-2}+1-d_{s-2}}=$ $m_{s-2, d_{s-2}}=\left|B_{s-2, d_{s-2}}\right|$. С другой стороны, так как $a_{l} \in B_{s-2, d_{s-2}}$, то $\left|B_{s-2, d_{s-2}}\right| \geqslant 1$. Поэтому возможны 2 подслучая.

1.1. Пусть $\left|B_{s-2, d_{s-2}}\right|=\left|B_{s-2, d_{s-2}^{\prime}}\right|=1$. Тогда $m_{s-2, d_{s-2}}=1, B_{s-2, d_{s-2}}=\left\{a_{l}\right\}$ и $B_{s-2, d_{s-2}^{\prime}}=\left\{a_{m}\right\}$. Отсюда, из определения множеств $B_{i, j}$ и из $(16),(18),(25)$ имеем

$$
l=\sum_{j=1}^{d_{s-2}-1} m_{s-2, j}+1=\sum_{j=1}^{d_{s-2}} m_{s-2, j},
$$

$$
\begin{gathered}
m=\sum_{j=1}^{d_{s-2}^{\prime}-1} m_{s-2, j}+1=2\left\lfloor\frac{n}{2}\right\rfloor-\sum_{j=d_{s-2}^{\prime}}^{2^{s-2}} m_{s-2, j}+1=2\left\lfloor\frac{n}{2}\right\rfloor-\sum_{j=d_{s-2}^{\prime}}^{2^{s-2}} m_{s-2,2^{s-2}-j+1}+1 \\
\quad=2\left\lfloor\frac{n}{2}\right\rfloor-\sum_{j=1}^{2^{s-2}-d_{s-2}^{\prime}+1} m_{s-2, j}+1=2\left\lfloor\frac{n}{2}\right\rfloor-\sum_{j=1}^{d_{s-2}} m_{s-2, j}+1=2\left\lfloor\frac{n}{2}\right\rfloor-l+1 .
\end{gathered}
$$

Далее, как нетрудно видеть из построения множества $A_{s, 1}$, данное множество обладает следующим свойством: для любого $j \in\left\{1, \ldots, 2\left\lfloor\frac{n}{2}\right\rfloor\right\}$ элементы $a_{j}$ и $a_{2\left\lfloor\frac{n}{2}\right\rfloor-j+1}$ либо одновременно принадлежат, либо одновременно не принадлежат множеству $A_{s, 1}$. Так как $m=2\left\lfloor\frac{n}{2}\right\rfloor-l+1$, то либо оба элемента $a_{l}, a_{m}$ принадлежат множеству $A_{s, 1}$, т. е. $B=\left\{a_{l}, a_{m}\right\} \subseteq A_{s, 1}$, либо они оба принадлежат множеству $A \backslash A_{s, 1}=A_{s, 2}$, т. е. $B \subseteq A_{s, 2}$. Однако обе этих альтернативы противоречат предположению о том, что для любого индекса $i_{1} \in\{1, \ldots, s\}$ одновременно $B \nsubseteq A_{i_{1}, 1}$ и $B \nsubseteq A_{i_{1}, 2}$. Случай 1.1 разобран.

1.2. Пусть $\left|B_{s-2, d_{s-2}}\right|=\left|B_{s-2, d_{s-2}^{\prime}}\right|=2$. Согласно определению множеств $B_{i, j}$, это означает, что $B_{s-2, d_{s-2}}=\left\{a_{p}, a_{p+1}\right\}, B_{s-2, d_{s-2}^{\prime}}=\left\{a_{q}, a_{q+1}\right\}$, где $2 \leqslant p+1 \leqslant 2\left\lfloor\frac{n}{2}\right\rfloor$ и $2 \leqslant q+1 \leqslant 2\left\lfloor\frac{n}{2}\right\rfloor$ в силу (20). Кроме того, так как $a_{l} \in B_{s-2, d_{s-2}}$ и $a_{m} \in B_{s-2, d_{s-2}^{\prime}}$, то $l \in\{p, p+1\}$ и $m \in\{q, q+1\}$. Если числа $l$ и $m$ имеют одинаковую чётность, то по построению множеств $A_{s-1,1}$ и $A_{s-1,2}$ оба элемента $a_{l}, a_{m}$ одновременно принадлежат либо $A_{s-1,1}$, либо $A_{s-1,2}$, т. е. либо $B \subseteq A_{s-1,1}$, либо $B \subseteq A_{s-1,2}$, однако обе этих альтернативы противоречат предположению о том, что для любого индекса $i_{1} \in\{1, \ldots, s\}$ одновременно $B \nsubseteq A_{i_{1}, 1}$ и $B \nsubseteq A_{i_{1}, 2}$. Таким образом, числа $l$ и $m$ имеют разную чётность. Далее, из определения множеств $B_{i, j},(16),(18),(25)$ и того, что $m_{s-2, d_{s-2}}=\left|B_{s-2, d_{s-2}}\right|=2$, имеем

$$
p=\sum_{j=1}^{d_{s-2}-1} m_{s-2, j}+1=\sum_{j=1}^{d_{s-2}} m_{s-2, j}+1-m_{s-2, d_{s-2}}=\sum_{j=1}^{d_{s-2}} m_{s-2, j}-1,
$$




$$
\begin{gathered}
q=\sum_{j=1}^{d_{s-2}^{\prime}-1} m_{s-2, j}+1=2\left\lfloor\frac{n}{2}\right\rfloor-\sum_{j=d_{s-2}^{\prime}}^{2^{s-2}} m_{s-2, j}+1=2\left\lfloor\frac{n}{2}\right\rfloor-\sum_{j=d_{s-2}^{\prime}}^{2^{s-2}} m_{s-2,2^{s-2}-j+1}+1 \\
=2\left\lfloor\frac{n}{2}\right\rfloor-\sum_{j=1}^{2^{s-2}-d_{s-2}^{\prime}+1} m_{s-2, j}+1=2\left\lfloor\frac{n}{2}\right\rfloor-\sum_{j=1}^{d_{s-2}} m_{s-2, j}+1=2\left\lfloor\frac{n}{2}\right\rfloor-p .
\end{gathered}
$$

Из последнего соотношения вытекает, что числа $p$ и $q$ имеют одинаковую чётность и $p+q=2\left\lfloor\frac{n}{2}\right\rfloor$. Так как $l \in\{p, p+1\}, m \in\{q, q+1\}$, числа $p$ и $q$ имеют одинаковую, а числа $l$ и $m-$ разную чётность, то либо $l=p$ и $m=q+1$, либо $l=p+1$ и $m=q$. В обоих случаях получаем соотношение $l+m=p+q+1=2\left\lfloor\frac{n}{2}\right\rfloor+1$, т. е. $m=2\left\lfloor\frac{n}{2}\right\rfloor-$ $l+1$. Последнее равенство совпадает с соответствующим равенством, полученным при разборе случая 1.1, и дальнейшие рассуждения проводятся аналогично этому случаю. Случай 1.2 , а вместе с ним случай 1 разобраны.

2. Пусть $n$ нечётно и $a_{n} \in B$. Без ограничения общности, $n=m$, т. е. $B=$ $\left\{a_{l}, a_{n}\right\}$. В этом случае из $(22)$ следует, что $a_{n} \in A_{i_{1}, 1}$ для любого $i_{1} \in\{1, \ldots, s-2\}$, а значит, $a_{n} \notin A_{i_{1}, 2}$. Так как по предположению $a_{l} \in A_{i_{1}, b_{i_{1}}}$ и $a_{n} \in A_{i_{1}, 3-b_{i_{1}}}$ для некоторого $b_{i_{1}} \in\{1 ; 2\}$, то $b_{i_{1}}=2$ и $a_{l} \in A_{i_{1}, 2}$ для любого $i_{1} \in\{1, \ldots, s-2\}$. Тогда при $i_{1}=1$ в силу (21) имеем $a_{l} \in A_{1,2}=B_{1,2}$. Если $s \geqslant 4$, то при $i_{1}=2$ имеем $a_{l} \in A_{2,2}$, а в таком случае $a_{l} \in B_{1,2} \cap A_{2,2}=B_{2,2 \cdot 2+2-2}=B_{2,2^{2}}$ в силу (23). Далее, если $s \geqslant 5$, то при $i_{1}=3$ имеем $a_{l} \in A_{3,2}$, а в таком случае $a_{l} \in B_{2,2^{2}} \cap A_{3,2}=B_{3,2^{2} \cdot 2+2-2}=B_{3,2^{3}}$ в силу (23). Рассуждая аналогично для $i_{1}=4,5, \ldots, s-2$, получим, что $a_{l} \in B_{s-2,2^{s-2}}$. По аналогии с разбором случая 2 получим, что $m_{s-2,1}=1$. В силу $(16),(19)$ имеем $\left|B_{s-2,2^{s-2}}\right|=m_{s-2,2^{s-2}}=m_{s-2,1}=1$, и единственный элемент множества $B_{s-2,2^{s-2}}$ по определению данного множества имеет номер

$$
\sum_{j=1}^{2^{s-2}-1} m_{s-2, j}+1=\sum_{j=1}^{2^{s-2}} m_{s-2, j}=2\left\lfloor\frac{n}{2}\right\rfloor
$$

в силу (18). Получаем, что $l=2\left\lfloor\frac{n}{2}\right\rfloor$ и $B=\left\{a_{2\left\lfloor\frac{n}{2}\right\rfloor}, a_{n}\right\}$. Из определения множества $A_{s-1,2}$ видно, что $a_{2\left\lfloor\frac{n}{2}\right\rfloor} \in A_{s-1,2}$ и $a_{n} \in A_{s-1,2}$, т. е. $B \subseteq A_{s-1,2}$. Но это противоречит тому, что для любого индекса $i_{1} \in\{1, \ldots, s\}$ выполнено соотношение $B \nsubseteq A_{i_{1}, 2}$. Случай 2 разобран. Свойство (4) множеств $A_{1,1}, A_{1,2}, \ldots, A_{s, 1}, A_{s, 2}$, а вместе с ним лемма 6 доказаны.

Вернёмся к доказательству основной теоремы, а именно, к получению оценки $L(f, N) \leqslant\left\lceil\log _{3}(2 N+1)\right\rceil$ в случае $n=1$ и $f\left(x_{1}\right)=\overline{x_{1}}$. Пусть $r=\left\lceil\log _{3}(2 N+1)\right\rceil$. Ясно, что $r \geqslant 1$. Опишем построение схем $S_{1}^{\prime \prime}, \ldots, S_{r}^{\prime \prime}$, которые составят проверяющий тест. В силу леммы 5 существуют такие целые неотрицательные числа $n_{i, j}$, $i=1, \ldots, r, j=1, \ldots, C_{r}^{i}$, что

$$
\sum_{i=1}^{r} \sum_{j=1}^{C_{r}^{i}} n_{i, j}=N
$$

причём

$$
n_{i, j} \leqslant 2^{i-1}
$$

для любых $i \in\{1, \ldots, r\}, j \in\left\{1, \ldots, C_{r}^{i}\right\}$ и

$$
n_{1,1}=n_{1,2}=\ldots=n_{1, r}=1 .
$$


Равенство (26) означает, что множество $\left\{E_{1}, \ldots, E_{N}\right\}$ может быть представлено в виде объединения таких попарно не пересекающихся множеств $V_{i, j}, i=1, \ldots, r$, $j=1, \ldots, C_{r}^{i}$, что для любых $i \in\{1, \ldots, r\}, j \in\left\{1, \ldots, C_{r}^{i}\right\}$ выполняется равенство

$$
\left|V_{i, j}\right|=n_{i, j} .
$$

(Ясно, что таких способов представления множества $\left\{E_{1}, \ldots, E_{N}\right\}$ в виде $\underset{\substack{i=1, \ldots, r \\ j=1, \ldots, C_{r}^{i}}}{\bigsqcup} V_{i, j}$ может быть много. Выберем из них для определённости какой-нибудь один, например, такой, при котором $V_{1,1}=\left\{E_{1}, \ldots, E_{n_{1,1}}\right\}$, а каждое следующее множество $V_{i, j}$ состоит из $n_{i, j}$ элементов с наименьшими номерами среди ещё не задействованных в предыдущих множествах.) Далее, каждому множеству $V_{i, j}, i=1, \ldots, r$, $j=1, \ldots, C_{r}^{i}$, поставим в соответствие двоичный набор $\tilde{\alpha}_{i, j}$ длины $r$, являющийся $j$-м в лексикографическом порядке среди всех двоичных наборов длины $r$, содержащих ровно $i$ единиц. Это можно сделать, так как число двоичных наборов длины $r$, содержащих ровно $i$ единиц, равно $C_{r}^{i}$. Будем считать, что каждый элемент из множества $V_{i, j}, i=1, \ldots, r, j=1, \ldots, C_{r}^{i}$, содержится в схеме $S_{q}^{\prime \prime}, q=1, \ldots, r$, тогда и только тогда, когда $q$-й (слева) разряд набора $\tilde{\alpha}_{i, j}$ равен 1 . Например, если $r=7$, $i=4$ и $j=3$, то $\tilde{\alpha}_{i, j}=(0,0,1,1,0,1,1)$ и каждый элемент из множества $V_{4,3}$ содержится в схемах $S_{3}^{\prime \prime}, S_{4}^{\prime \prime}, S_{6}^{\prime \prime}, S_{7}^{\prime \prime}$ и не содержится в схемах $S_{1}^{\prime \prime}, S_{2}^{\prime \prime}, S_{5}^{\prime \prime}$. Таким образом, для каждой из схем $S_{1}^{\prime \prime}, \ldots, S_{r}^{\prime \prime}$ определены все элементы, которые в ней содержатся. А именно, в каждой схеме $S_{q}^{\prime \prime}, q=1, \ldots, r$, содержатся в точности все элементы из всех таких множеств $V_{i, j}$, что $q$-й разряд набора $\tilde{\alpha}_{i, j}$ равен 1 . Множество элементов, содержащихся в схеме $S_{q}^{\prime \prime}, q=1, \ldots, r$, обозначим через $M_{q}$.

Определим теперь строение каждой схемы $S_{q}^{\prime \prime}, q=1, \ldots, r$. Так как $n=1$ по предположению случая 4 , то каждый элемент из множества $M_{q}$ имеет один вход. Будем предполагать, что схема $S_{q}^{\prime \prime}$ представляет собой цепь, содержащую все элементы из множества $M_{q}$, на вход верхнего элемента которой подаётся переменная $x$, а выходом схемы $S_{q}^{\prime \prime}$ является выход нижнего элемента этой цепи. Определим порядок следования элементов из множества $M_{q}$ в этой цепи. Для этого воспользуемся некоторыми вспомогательными построениями. Рассмотрим каждое непустое множество $V_{i, j}, i=1, \ldots, r, j=1, \ldots, C_{r}^{i}$. Тогда $n_{i, j}=\left|V_{i, j}\right| \geqslant 1$ в силу (29) и можно определить число $s_{i, j}=\left\lceil\log _{2} n_{i, j}\right\rceil+1$. Применяя лемму 6 для $A=V_{i, j}, n=n_{i, j}$ и $s=s_{i, j}$, получим, что существуют такие $2 s_{i, j}$ множеств $V_{1,1}^{i, j}, V_{1,2}^{i, j}, \ldots, V_{s_{i, j}, 1}^{i, j}, V_{s_{i, j}, 2}^{i, j}$, что для любого $t \in\left\{1, \ldots, s_{i, j}\right\}$ выполнены соотношения

$$
\begin{gathered}
|| V_{t, 1}^{i, j}|-| V_{t, 2}^{i, j}|| \leqslant 2, \\
V_{t, 1}^{i, j} \sqcup V_{t, 2}^{i, j}=V_{i, j}
\end{gathered}
$$

и, кроме того, справедливо следующее утверждение $(* * *)$ : для любого двухэлементного подмножества $B_{i, j}$ множества $V_{i, j}$ существуют такие индексы $t_{1}, t_{2} \in$ $\left\{1, \ldots, s_{i, j}\right\}$, что $B_{i, j} \subseteq V_{t_{1}, 1}^{i, j}$ или $B_{i, j} \subseteq V_{t_{1}, 2}^{i, j}$ и при этом $B_{i, j} \nsubseteq V_{t_{2}, 1}^{i, j}, B_{i, j} \nsubseteq V_{t_{2}, 2}^{i, j}$. (Оценка (30) является более грубой, чем совокупность свойств 1), 2) из условия леммы 6 , но нам её будет достаточно.) Далее, из (27) и определения числа $s_{i, j}$ следует, что

$$
i \geqslant\left\lceil\log _{2} n_{i, j}\right\rceil+1=s_{i, j} .
$$

В случае $i>s_{i, j}$ положим $V_{s_{i, j}+1,1}^{i, j}=\ldots=V_{i, 1}^{i, j}=V_{1,1}^{i, j}, V_{s_{i, j}+1,2}^{i, j}=\ldots=V_{i, 2}^{i, j}=V_{1,2}^{i, j}$. Таким образом, определены $2 i$ множеств $V_{1,1}^{i, j}, V_{1,2}^{i, j}, \ldots, V_{i, 1}^{i, j}, V_{i, 2}^{i, j}$, причём соотношения (30), (31) верны не только для любого $t \in\left\{1, \ldots, s_{i, j}\right\}$, но и - в случае $i>s_{i, j}$ 
- для $t=s_{i, j}+1, \ldots, i$. Действительно, для $t=s_{i, j}+1, \ldots, i$ имеем

$$
\begin{gathered}
|| V_{t, 1}^{i, j}|-| V_{t, 2}^{i, j}||=|| V_{1,1}^{i, j}|-| V_{1,2}^{i, j}|| \leqslant 2, \\
V_{t, 1}^{i, j} \sqcup V_{t, 2}^{i, j}=V_{1,1}^{i, j} \sqcup V_{1,2}^{i, j}=V_{i, j} .
\end{gathered}
$$

Получаем, что соотношения (30), (31) верны для всех $t=1, \ldots, i$.

Введём для удобства функцию $t(i, j, q)$, определённую на всех таких тройках $(i, j, q)$, что $i \in\{1, \ldots, r\}, j \in\left\{1, \ldots, C_{r}^{i}\right\}, q \in\{1, \ldots, r\}$, и равную

$$
\begin{cases}0, & \text { если } q \text {-й (слева) разряд набора } \tilde{\alpha}_{i, j} \text { равен } 0, \\ p, & \text { если } q \text {-й разряд набора } \tilde{\alpha}_{i, j} \text { является } p \text {-м слева единичным разрядом } \\ & \text { этого набора. }\end{cases}
$$

Например, если $\tilde{\alpha}_{i, j}=(0,0,1,1,0,1,1)$, то $t(i, j, 1)=t(i, j, 2)=t(i, j, 5)=0, t(i, j, 3)=$ $1, t(i, j, 4)=2, t(i, j, 6)=3, t(i, j, 7)=4$. Отметим, что всегда $t(i, j, q) \leqslant i$, так как в наборе $\tilde{\alpha}_{i, j}$ содержится ровно $i$ единиц. Тогда каждое множество $M_{q}, q=1, \ldots, r$, как следует из определения этого множества и (31), представимо в виде

$$
M_{q}=\bigsqcup_{(i, j): t(i, j, q)>0} V_{i, j}=\bigsqcup_{\begin{array}{c}
(i, j): t(i, j, q)>0, \\
V_{i, j} \text { непусто }
\end{array}} V_{i, j}=\bigsqcup_{\begin{array}{c}
(i, j): t(i, j, q)>0, \\
V_{i, j} \text { непусто }
\end{array}}\left(V_{t(i, j, q), 1}^{i, j} \sqcup V_{t(i, j, q), 2}^{i, j}\right) .
$$

Разобьём множество $M_{q}$ на два непересекающихся множества $M_{q, 1}$ и $M_{q, 2}$ следующим образом. Из каждой пары множеств $V_{t(i, j, q), 1}^{i, j}, V_{t(i, j, q), 2}^{i, j}$, стоящих в правой части представления (33), все элементы одного множества отнесём ко множеству $M_{q, 1}$, а все элементы другого - ко множеству $M_{q, 2}$ так, чтобы для полученных в итоге множеств $M_{q, 1}$ и $M_{q, 2}$ выполнялось неравенство

$$
|| M_{q, 1}|-| M_{q, 2}|| \leqslant 1 \text {. }
$$

Покажем, что это можно сделать. Изначально будем считать, что множества $M_{q, 1}$ и $M_{q, 2}$ пусты. Заметим, что в правую часть представления (33) входят множества $V_{t(1, r-q+1, q), 1}^{1, r-q+1}$ и $V_{t(1, r-q+1, q), 2}^{1, r-q+1}$. Действительно, так как $q \in\{1, \ldots, r\}$, то $r-q+1 \in\{1, \ldots, r\}$ и множество $V_{1, r-q+1}$ определено; из определений наборов $\tilde{\alpha}_{i, j}$ следует, что $\tilde{\alpha}_{1, r-q+1}=(\underbrace{0, \ldots, 0}_{q-1}, 1, \underbrace{0, \ldots, 0}_{r-q})$, а из определения функции $t(i, j, q)$ - что $t(1, r-q+1, q)=1>0$; наконец, из (28), (29) следует, что $\left|V_{1, r-q+1}\right|=n_{1, r-q+1}=1$ и множество $V_{1, r-q+1}$ не пусто. Из последнего соотношения и равенства $V_{1, r-q+1}=V_{t(1, r-q+1, q), 1}^{1, r-q+1} \sqcup V_{t(1, r-q+1, q), 2}^{1, r-q+1} \quad$ (согласно (31)) следует, что одно из множеств $V_{t(1, r-q+1, q), 1}^{1, r-q+1}, V_{t(1, r-q+1, q), 2}^{1, r-q+1}$ пусто, а в другом содержится ровно один элемент. Отнесём этот элемент ко множеству $M_{q, 1}$ и будем считать, что пара множеств $V_{t(1, r-q+1, q), 1}^{1, r-q+1}, V_{t(1, r-q+1, q), 2}^{1, r-q+1}$ уже рассмотрена. На данном этапе $\left|M_{q, 1}\right|=1$, $\left|M_{q, 2}\right|=0$ и поэтому

$$
\left|M_{q, 1}\right|-\left|M_{q, 2}\right|=1
$$

Далее, все не рассмотренные пары множеств $V_{t(i, j, q), 1}^{i, j}, V_{t(i, j, q), 2}^{i, j}$, стоящие в правой части представления (33), разобьём на два класса. К первому классу отнесём все пары, для которых выполняется равенство

$$
|| V_{t(i, j, q), 1}^{i, j}|-| V_{t(i, j, q), 2}^{i, j}||=2,
$$


а ко второму - все остальные пары. В силу (30) для каждой пары из второго класса выполняется неравенство

$$
|| V_{t(i, j, q), 1}^{i, j}|-| V_{t(i, j, q), 2}^{i, j}|| \leqslant 1
$$

Рассмотрим произвольную пару $\left(V_{t(i, j, q), 1}^{i, j}, V_{t(i, j, q), 2}^{i, j}\right)_{1}$ из первого класса, если такая пара существует. Отнесём все элементы из того множества, входящего в эту пару, в котором меньше элементов, ко множеству $M_{q, 1}$, а все элементы из другого множества из этой пары - ко множеству $M_{q, 2}$. Тогда в силу $(35),(36)$ для полученных множеств $M_{q, 1}$ и $M_{q, 2}$ выполняется равенство

$$
\left|M_{q, 2}\right|-\left|M_{q, 1}\right|=1
$$

Затем рассмотрим любую другую пару $\left(V_{t(i, j, q), 1}^{i, j}, V_{t(i, j, q), 2}^{i, j}\right)_{2}$ из первого класса, если такая пара существует. Отнесём все элементы из того множества, входящего в эту пару, в котором меньше элементов, ко множеству $M_{q, 2}$, а все элементы из другого множества из этой пары - ко множеству $M_{q, 1}$. Тогда в силу $(36),(38)$ для полученных множеств $M_{q, 1}$ и $M_{q, 2}$ выполняется равенство (35). Далее рассмотрим любую пару $\left(V_{t(i, j, q), 1}^{i, j}, V_{t(i, j, q), 2}^{i, j}\right)_{2}$ из первого класса, отличную от ранее рассмотренных, если такая пара существует. Отнесём все элементы из того множества, входящего в эту пару, в котором меньше элементов, ко множеству $M_{q, 1}$, а все элементы из другого множества из этой пары - ко множеству $M_{q, 2}$. Тогда в силу $(35),(36)$ для полученных множеств $M_{q, 1}$ и $M_{q, 2}$ выполняется равенство (38). Действуя и далее аналогичным образом, получим, что все элементы одного из множеств каждой пары из первого класса отнесены ко множеству $M_{q, 1}$, а все элементы другого множества той же пары - ко множеству $M_{q, 2}$, причём для полученных множеств выполнено одно из неравенств (35), (38), а значит - и неравенство (34).

Далее будем последовательно рассматривать пары множеств из второго класса (если такие пары существуют) и относить все элементы одного из множеств рассматриваемой пары ко множеству $M_{q, 1}$, а все элементы другого множества этой же пары - ко множеству $M_{q, 2}$, следя только за тем, чтобы после каждой такой операции не нарушалось неравенство (34). Для этого достаточно следить, чтобы в случае обращения обоих неравенств (34), (37) в равенства при рассмотрении очередной пары все элементы из того её множества, в котором меньше элементов, были отнесены к тому из множеств $M_{q, 1}, M_{q, 2}$, в котором больше элементов, и наоборот. В случае же равенства нулю хотя бы одного из выражений, стоящих в левых частях неравенств (34), (37), после выполнения очередного шага неравенство (34), как нетрудно видеть, будет выполняться вне зависимости от того, элементы какого множества из рассматриваемой пары мы относим к $M_{q, 1}$, а какого - к $M_{q, 2}$.

В результате указанной процедуры множество $M_{q}$ в силу (33) разбивается на два непересекающихся множества $M_{q, 1}$ и $M_{q, 2}$, причём для каждой пары множеств $V_{t(i, j, q), 1}^{i, j}, V_{t(i, j, q), 2}^{i, j}$ стоящих в правой части представления (33), все элементы одного из этих множеств принадлежат $M_{q, 1}$, а все элементы другого $-M_{q, 2}$, и выполнено неравенство (34). 
Пусть

$$
\begin{array}{r}
M_{q}^{\prime}= \begin{cases}M_{q, 1}, & \text { если }\left|M_{q, 1}\right| \geqslant\left|M_{q, 2}\right|, \\
M_{q, 2}, & \text { если }\left|M_{q, 1}\right|<\left|M_{q, 2}\right|,\end{cases} \\
M_{q}^{\prime \prime}= \begin{cases}M_{q, 2}, & \text { если }\left|M_{q, 1}\right| \geqslant\left|M_{q, 2}\right|, \\
M_{q, 1}, & \text { если }\left|M_{q, 1}\right|<\left|M_{q, 2}\right| .\end{cases}
\end{array}
$$

Тогда неупорядоченные пары $\left(M_{q, 1}, M_{q, 2}\right)$ и $\left(M_{q}^{\prime}, M_{q}^{\prime \prime}\right)$ совпадают и $\left|M_{q}^{\prime}\right| \geqslant\left|M_{q}^{\prime \prime}\right|$, а отсюда и из (34) следует, что $0 \leqslant\left|M_{q}^{\prime}\right|-\left|M_{q}^{\prime \prime}\right| \leqslant 1$, что в совокупности с соотношением $\left|M_{q}\right|=\left|M_{q, 1}\right|+\left|M_{q, 2}\right|=\left|M_{q}^{\prime}\right|+\left|M_{q}^{\prime \prime}\right|$ приводит к равенствам

$$
\begin{aligned}
& \left|M_{q}^{\prime}\right|=\left\lceil\frac{\left|M_{q}\right|}{2}\right\rceil, \\
& \left|M_{q}^{\prime \prime}\right|=\left\lfloor\frac{\left|M_{q}\right|}{2}\right\rfloor .
\end{aligned}
$$

Определим порядок следования элементов из множества $M_{q}$ в схеме $S_{q}^{\prime \prime}, q=1, \ldots, r$. Напомним, что схема $S_{q}^{\prime \prime}$ представляет собой цепь элементов, на вход верхнего элемента которой подаётся переменная $x$, а выходом схемы $S_{q}^{\prime \prime}$ является выход нижнего элемента этой цепи. Будем говорить, что функциональный элемент расположен в схеме $S_{q}^{\prime \prime}$ на нечётном месте, если ниже его в указанной цепи расположено чётное число элементов. (Таковым, например, является выходной элемент схемы $S_{q}^{\prime \prime}$.) Все остальные элементы в этой схеме будем считать расположенными на чётных местах. Так как общее число элементов в схеме $S_{q}^{\prime \prime}$ равно $\left|M_{q}\right|$, то легко видеть, что в ней имеется ровно $\left\lceil\frac{\left|M_{q}\right|}{2}\right\rceil$ нечётных и ровно $\left\lfloor\frac{\left|M_{q}\right|}{2}\right\rfloor$ чётных мест. Отсюда и из $(39),(40)$ заключаем, что все элементы из множества $\left|M_{q}^{\prime}\right|$ могут быть размещены на нечётных, а все элементы из множества $\left|M_{q}^{\prime \prime}\right|$ - на чётных местах. (И даже существует $\left(\left\lceil\frac{\left|M_{q}\right|}{2}\right\rceil\right) ! \cdot\left(\left\lfloor\frac{\left|M_{q}\right|}{2}\right\rfloor\right) !$ способов это сделать. Из них для определённости можно выбрать какой-нибудь один, например - в порядке возрастания номеров элементов, если двигаться по схеме $S_{q}^{\prime \prime}$ сверху вниз.)

В результате все схемы $S_{1}^{\prime \prime}, \ldots, S_{r}^{\prime \prime}$ полностью определены. Из вида этих схем и того, что $f\left(x_{1}\right)=\overline{x_{1}}$, непосредственно вытекает

Лемма 7. Пусть имеет место некоторая неисправность системы элементов и $q \in\{1, \ldots, r\}$. Тогда функиия, реализуемая схемой $S_{q}^{\prime \prime}$, равна

$$
\begin{cases}x \text { или } \bar{x}, & \text { если все элементы в схеме } S_{q}^{\prime \prime} \text { исправны; } \\ \delta, & \text { если некоторый элемент, расположенный в схеме } S_{q}^{\prime \prime} \text { на нечётном } \\ & \text { месте, неисправен и выдаёт константу } \delta ; \\ \bar{\delta}, & \text { если некоторый элемент, расположенный в схеме } S_{q}^{\prime \prime} \text { на чётном } \\ & \text { месте, неисправен и выдаёт константу } \delta .\end{cases}
$$

Лемма 8. Набор $\left(S_{1}^{\prime \prime}, \ldots, S_{r}^{\prime \prime}\right)$ является проверяющим тестом.

Доказательство. Пусть $H_{1}$ и $H_{2}$ - две произвольные неисправности системы элементов, при которых множества неисправных элементов различны. Надо доказать, что наборы функций, реализуемых схемами $S_{1}^{\prime \prime}, \ldots, S_{r}^{\prime \prime}$ при этих двух неисправностях, не совпадают. Предположим сначала, что при одной из неисправностей $H_{1}$ и 
$H_{2}$ (без ограничения общности, при $H_{1}$ ) все элементы исправны. Тогда при неисправности $H_{2}$ некоторый элемент $E_{a}$ неисправен. Согласно описанным выше построениям, элемент $E_{a}$ принадлежит некоторому множеству $V_{i, j}$ (где $i \geqslant 1$ ), которому соответствует двоичный набор $\tilde{\alpha}_{i, j}$ длины $r$, содержащий ровно $i$ единиц, а это означает, что данный элемент содержится ровно в $i$ схемах из $S_{1}^{\prime \prime}, \ldots, S_{r}^{\prime \prime}$. Тогда в силу леммы 7 каждая из этих $i$ схем реализует одну из функций $x, \bar{x}$ при неисправности $H_{1}$ и некоторую булеву константу при неисправности $H_{2}$, откуда следует, что наборы функций, реализуемых схемами $S_{1}^{\prime \prime}, \ldots, S_{r}^{\prime \prime}$ при этих двух неисправностях, не совпадают.

Пусть теперь при неисправности $H_{1}$ неисправен некоторый элемент $E_{a_{1}}$, а при неисправности $H_{2}$ - некоторый элемент $E_{a_{2}}$. Тогда $a_{1} \neq a_{2}$. Согласно описанным выше построениям, элементы $E_{a_{1}}$ и $E_{a_{2}}$ принадлежит некоторым множествам соответственно $V_{i_{1}, j_{1}}$ и $V_{i_{2}, j_{2}}$. Возможны 2 случая.

1. Выполняется хотя бы одно из неравенств $i_{1} \neq i_{2}, j_{1} \neq j_{2}$. Тогда двоичные наборы $\tilde{\alpha}_{i_{1}, j_{1}}$ и $\tilde{\alpha}_{i_{2}, j_{2}}$ длины $r$ различаются (это следует из определения наборов $\tilde{\alpha}_{i, j}$ ). Пусть они различаются в $q$-м разряде. Без ограничения общности, в $q$-м разряде набора $\tilde{\alpha}_{i_{1}, j_{1}}$ стоит единица, а в $q$-м разряде набора $\tilde{\alpha}_{i_{2}, j_{2}}-$ нуль. Тогда, согласно построению схемы $S_{q}^{\prime \prime}$, в ней содержатся все элементы из множества $V_{i_{1}, j_{1}}$, в частности, элемент $E_{a_{1}}$, и не содержится ни одного элемента из множества $V_{i_{2}, j_{2}}$, в частности, элемента $E_{i_{2}}$. Тогда в силу леммы 7 схема $S_{q}^{\prime \prime}$ реализует некоторую булеву константу при неисправности $H_{1}$ и одну из функций $x, \bar{x}$ при неисправности $H_{2}$, откуда следует, что наборы функций, реализуемых схемами $S_{1}^{\prime \prime}, \ldots, S_{r}^{\prime \prime}$ при этих двух неисправностях, не совпадают. Случай 1 разобран.

2. Одновременно $i_{1}=i_{2}$ и $j_{1}=j_{2}$. Пусть $i=i_{1}, j=j_{1}$. Тогда оба элемента $E_{a_{1}}$, $E_{a_{2}}$ принадлежат множеству $V_{i, j}$. В силу утверждения $(* * *)$ для множества $B=$ $\left\{E_{a_{1}}, E_{a_{2}}\right\}$ существуют такие индексы $t_{1}, t_{2} \in\left\{1, \ldots, s_{i, j}\right\}$, что $B \subseteq V_{t_{1}, 1}^{i, j}$ или $B \subseteq V_{t_{1}, 2}^{i, j}$ и при этом $B \nsubseteq V_{t_{2}, 1}^{i, j}, B \nsubseteq V_{t_{2}, 2}^{i, j}$. Из последних двух соотношений, поскольку $B \subseteq V_{i, j}$ и $V_{t_{2}, 1}^{i, j} \sqcup V_{t_{2}, 2}^{i, j}=V_{i, j}$ в силу (31), заключаем, что либо $E_{a_{1}} \in V_{t_{2}, 1}^{i, j}$ и $E_{a_{2}} \in V_{t_{2}, 2}^{i, j}$, либо $E_{a_{1}} \in V_{t_{2}, 2}^{i, j}$ и $E_{a_{2}} \in V_{t_{2}, 1}^{i, j}$. Далее, в силу (32) имеем $t_{1}, t_{2} \in\{1, \ldots, i\}$. Так как в наборе $\tilde{\alpha}_{i, j}$ содержится ровно $i$ единиц, то в силу определения функции $t(i, j, q)$ существуют такие числа $q_{1}, q_{2} \in\{1, \ldots, r\}$, что $t\left(i, j, q_{1}\right)=t_{1}$ и $t\left(i, j, q_{2}\right)=t_{2}$. Отсюда и в силу равенства (33) и того, что множество $V_{i, j}$ непусто, имеем $V_{t_{1}, 1}^{i, j}=V_{t\left(i, j, q_{1}\right), 1}^{i, j} \subseteq M_{q_{1}}$, $V_{t_{1}, 2}^{i, j}=V_{t\left(i, j, q_{1}\right), 2}^{i, j} \subseteq M_{q_{1}}, V_{t_{2}, 1}^{i, j}=V_{t\left(i, j, q_{2}\right), 1}^{i, j} \subseteq M_{q_{2}}, V_{t_{2}, 2}^{i, j}=V_{t\left(i, j, q_{2}\right), 2}^{i, j} \subseteq M_{q_{2}}$. В силу этих соотношений, с учётом построения множеств $M_{q, 1}, M_{q, 2}$ и определения множеств $M_{q}^{\prime}$, $M_{q}^{\prime \prime}$ для каждого $q=1, \ldots, r$, можно утверждать, что либо $V_{t_{1}, 1}^{i, j} \subseteq M_{q_{1}}^{\prime}$ и $V_{t_{1}, 2}^{i, j} \subseteq M_{q_{1}}^{\prime \prime}$, либо $V_{t_{1}, 1}^{i, j} \subseteq M_{q_{1}}^{\prime \prime}$ и $V_{t_{1}, 2}^{i, j} \subseteq M_{q_{1}}^{\prime}$ и, кроме того, либо $V_{t_{2}, 1}^{i, j} \subseteq M_{q_{2}}^{\prime}$ и $V_{t_{2}, 2}^{i, j} \subseteq M_{q_{2}}^{\prime \prime}$, либо $V_{t_{2}, 1}^{i, j} \subseteq M_{q_{2}}^{\prime \prime}$ и $V_{t_{2}, 2}^{i, j} \subseteq M_{q_{2}}^{\prime}$. Учитывая написанные выше соотношения для множества $B$ и элементов $E_{a_{1}}, E_{a_{2}}$, получаем, что либо $B \subseteq M_{q_{1}}^{\prime}$, либо $B \subseteq M_{q_{1}}^{\prime \prime}$; кроме того, либо $E_{a_{1}} \in M_{q_{2}}^{\prime}$ и $E_{a_{2}} \in M_{q_{2}}^{\prime \prime}$, либо $E_{a_{1}} \in M_{q_{2}}^{\prime \prime}$ и $E_{a_{2}} \in M_{q_{2}}^{\prime}$. Так как в схеме $S_{q_{1}}^{\prime \prime}$ все элементы из множества $M_{q_{1}}^{\prime}$ расположены на нечётных, а все элементы из множества $M_{q_{1}}^{\prime \prime}$ - на чётных местах, то в этой схеме элементы $E_{a_{1}}$ и $E_{a_{2}}$ расположены на местах одной чётности. Так как в схеме $S_{q_{2}}^{\prime \prime}$ все элементы из множества $M_{q_{2}}^{\prime}$ расположены на нечётных, а все элементы из множества $M_{q_{2}}^{\prime \prime}$ - на чётных местах, то в этой схеме элементы $E_{a_{1}}$ и $E_{a_{2}}$ расположены на местах разной чётности. Пусть $H_{1}=\left\{\left(E_{a_{1}}, \delta_{1}\right)\right\}, H_{2}=\left\{\left(E_{a_{2}}, \delta_{2}\right)\right\}$, где $\delta_{1}, \delta_{2} \in\{0 ; 1\}$. Рассмотрим 2 подслучая. 
2.1. Пусть $\delta_{1} \neq \delta_{2}$. Тогда в силу вышенаписанного и леммы 7 схема $S_{q_{1}}^{\prime \prime}$ реализует при неисправностях $H_{1}$ и $H_{2}$ различные булевы константы (либо соответственно $\delta_{1}$ и $\delta_{2}$, либо соответственно $\overline{\delta_{1}}$ и $\overline{\delta_{2}}$ ), откуда следует, что наборы функций, реализуемых схемами $S_{1}^{\prime \prime}, \ldots, S_{r}^{\prime \prime}$ при этих двух неисправностях, не совпадают. Случай 2.1 разобран.

2.2. Пусть $\delta_{1}=\delta_{2}$. Тогда в силу вышенаписанного и леммы 7 схема $S_{q_{2}}^{\prime \prime}$ реализует при неисправностях $H_{1}$ и $H_{2}$ различные булевы константы (либо соответственно $\delta_{1}$ и $\overline{\delta_{2}}$, либо соответственно $\overline{\delta_{1}}$ и $\delta_{2}$ ), откуда следует, что наборы функций, реализуемых схемами $S_{1}^{\prime \prime}, \ldots, S_{r}^{\prime \prime}$ при этих двух неисправностях, не совпадают. Случай 2.2 разобран. Лемма 8 доказана.

Из леммы 8 получаем соотношение $L(f, N) \leqslant r=\left\lceil\log _{3}(2 N+1)\right\rceil$. Случай 4 разобран.

Теорема доказана.

В заключение автор выражает глубокую благодарность своему научному руководителю профессору Н. П. Редькину за постановку задачи и внимание к работе.

\section{Список литературы}

1. Лупанов О. Б., Асимптотические оценки сложности управляющих систем, Издательство МГУ, Москва, 1984.

2. Попков К. А., "Оценки длин проверяющих и диагностических тестов для функциональных элементов", Дискретный анализ и исследование операций, 21:6 (2014), 73-89.

3. Попков К. А., "Проверяющие и диагностические тесты для конъюнкторов, дизъюнкторов и инверторов", Вестник Московского университета. Серия 1. Математика. Механика, 2014, №6, 40-44. 